\title{
PDF modeling of a bluff-body stabilized turbulent flame
}

\author{
M. Muradoglu ${ }^{\mathrm{a}}$, K. Liu ${ }^{\mathrm{b}}$, S.B. Pope ${ }^{\mathrm{b}, *}$ \\ ${ }^{a}$ Department of Mechanical Engineering, Koc University, Rumelifeneri Yolu, Sariyer, 80910, Istanbul, Turkey \\ ${ }^{\mathrm{b}}$ Sibley School of Mechanical and Aerospace Engineering, Cornell University, Ithaca, NY 14853, USA
}

Received 11 January 2002; received in revised form 22 June 2002; accepted 11 July 2002

\begin{abstract}
The velocity-turbulent frequency-compositions PDF method combined with the consistent hybrid finite volume (FV)/particle solution algorithm is applied to a bluff-body stabilized turbulent flame. The statistical stationarity is shown and the performance of the PDF method is assessed by comparing the mean fields with the available experimental data. The effects of the model constants $C_{\omega 1}$ in the turbulence frequency model and $C_{\phi}$ in the mixing model on the numerical solutions are examined and it is found that all the mean fields are very sensitive to the changes in $C_{\omega 1}$ while only the mixture fraction variance seems to be very sensitive to the changes in $C_{\phi}$ but not the other mean fields. The spatial and bias errors are also examined and it is shown that the hybrid method is second order accurate in space and the bias error is vanishingly small in all the mean fields. The grid size and the number of particles per cell are determined for a 5\% error tolerance. The chemistry is described by the simplest possible flamelet/PDF model. Hence the main focus of the paper is on the accurate calculations of the mean flow, turbulence and mixing, which lays the foundation for future work in which the chemistry is described in greater detail. () 2003 The Combustion Institute. All rights reserved.
\end{abstract}

Keywords:Turbulent combustion; PDF methods

\section{Introduction}

The probability density function (PDF) method has proven to be among the most promising approaches for accurate modeling of turbulent reacting flows of practical importance [1-3]. Compared to conventional turbulence models, the PDF method offers the unique advantages of being able to take into account the important processes of convection and non-linear reaction in closed form [4]. Hence the effects of turbulent fluctuations on chemical reactions are treated exactly, and the gradient diffusion assumption is avoided. Of these advantages, the exact treatment of finite-rate non-linear chemistry makes

\footnotetext{
* Corresponding author. Tel.: +1-607-255-4314; fax: $+1-607-255-1222$.

E-mail address: pope@mae.cornell.edu (S.B. Pope).
}

the PDF method particularly attractive for simulations of complex turbulent reacting flows.

As for any turbulence model, an efficient numerical solution algorithm is of essential importance to apply the PDF method to flow problems of practical interest. Significant progress has been recently made in this direction by the development of the consistent hybrid finite volume (FV)/particle-based Monte Carlo method $[5,6]$. The hybrid method combines the best features of the FV and particle-based Monte Carlo methods to efficiently solve the PDF model equations. The method is completely consistent at the level of governing equations and the full consistency at the numerical solution level is enforced by using efficient correction algorithms [6]. It has been shown that the consistent hybrid method is computationally more efficient than the best available alternative solution technique by a factor of an order of magnitude or more $[2,6]$. The numerical efficiency of the hybrid 
algorithm is further improved by the recent development of a local time stepping algorithm that has been shown to accelerate the global convergence of the hybrid method as much as by a factor of 10 depending on grid stretching [7]. Combined with the local time stepping algorithm, the consistent hybrid method thus makes the PDF methodology a feasible design tool for the practical applications in engineering or elsewhere. The complete descriptions of the consistent hybrid method and the local time stepping algorithm can be found in the references $[5,6]$ and [7], respectively.

The primary purpose of the present work is to demonstrate the performance of the velocity-turbulent frequency-compositions joint PDF method [8] combined with the consistent hybrid FV/particle solution algorithm in predicting the properties of the bluff-body stabilized turbulent flame studied experimentally first by Dally et al. [9] and recently by Kalt and Masri [10]. The bluff-body flames have received significant attention in recent years because of their relevance to many engineering applications such as bluff-body combustors widely used in industrial applications due to the enhanced mixing characteristics, improved flame stability and ease of combustion control [11]. Besides its practical importance, the bluffbody flame studied here provides an excellent but challenging test case for turbulence and chemistry models as well as for numerical solution algorithms for studying turbulence and chemistry interactions in turbulent recirculating flows due to its simple and well defined initial and boundary conditions, and its ability to maintain the flame stabilization for a wide range of inlet flow conditions with a complex recirculation zone.

In this work the chemistry is described by the simplest possible flamelet/PDF model. The composition is assumed to be uniquely related to the mixture fraction in the same way as in a mildly strained laminar flame; and the mixture fraction is included in the joint PDF which is calculated. Hence, the focus here is on the accurate calculation of the mean flow, turbulence, and mixing. This lays the foundation for future work in which the chemistry is described in greater detail, as for example, the piloted jet flame studies of Xu and Pope [12] and Tang et al. [13].

Along with the experimental study, Dally et al. [9] also performed numerical simulations of the bluffbody flame using the standard and modified $k-\epsilon$ and Reynolds stress turbulence models. The same flame has also been modeled using a conditional moment closure (CMC) model by Kim et al. [14] and a cubic $k-\epsilon$ model by Merci et al. [15]. Similar bluff-body stabilized flames with various geometrical configurations and fuels have also been investigated numerically in the literature using different ap- proaches including the standard $k-\epsilon$ model [16], Reynolds stress models [16], the conditional moment closure model [17], PDF models [16,18], and large eddy simulation (LES) [19].

In the next section, the PDF modeling and the numerical solution algorithm are briefly reviewed. The model equations are presented in the context of the consistent hybrid method. The numerical solution procedure and the time-averaging algorithm are also described in this section. The test case of the bluffbody stabilized turbulent flame is briefly described, and the present PDF calculations are compared with the experimental data, in the Section 3, where the sensitivities of the numerical solutions to the model constants are also presented. Finally, conclusions are drawn in Section 4.

\section{PDF modeling and solution algorithm}

The velocity-turbulent frequency-compositions joint PDF model provides a complete closure for turbulent reacting flows [1]. At position $x$ and time $t$, the mass-weighted joint $\operatorname{PDF} \tilde{f}(\mathbf{V}, \boldsymbol{\psi}, \theta ; \mathbf{x}, t)$ is defined as the probability density function of the simultaneous event $\mathbf{U}(\mathbf{x}, t)=\mathbf{V}, \boldsymbol{\phi}(\mathbf{x}, t)=\boldsymbol{\psi}$, and $\omega(\mathbf{x}, t)=\theta$, where $\mathbf{U}, \boldsymbol{\phi}$, and $\omega$ are the velocity vector, the set of compositions and the turbulent frequency, respectively, and $\mathbf{V}, \boldsymbol{\psi}$, and $\theta$ are independent sample space variables in the velocity-turbulent frequency-compositions space. Two basic properties of $\tilde{f}$ are

$\int \tilde{f}(\mathbf{V}, \boldsymbol{\psi}, \theta ; \mathbf{x}, t) d \mathbf{V} d \boldsymbol{\psi} d \theta=1$,

and

$\int Q(\mathbf{V}, \boldsymbol{\psi}, \theta ; \mathbf{x}, t) \tilde{f}(\mathbf{V}, \boldsymbol{\psi}, \theta ; \mathbf{x}, t) d \mathbf{V} d \boldsymbol{\psi} d \theta=\tilde{Q}$,

$Q$ is a random variable and $\tilde{Q}$ is by definition the density-weighted (Favre-averaged) mean of $Q$. In the present PDF approach, the exact transport equation for $\tilde{f}$ is derived from the Navier-Stokes equations and the unclosed terms are modeled by constructing stochastic differential equations governing selected flow properties as briefly discussed in the following section. A detailed discussion about the present PDF method can be found in $[1,4]$.

\subsection{Model equations}

The model equations are presented in the context of the consistent hybrid method [5]. In the hybrid 
approach, the mean conservation equations for mass, momentum, and energy are directly derived from the modeled joint PDF transport equation, and are coupled with the mean equation of state. The mean conservation equations are then solved by a FV method to obtain the mean density, mean velocity, and mean equivalent sensible internal energy fields, and the pressure field is subsequently obtained from the mean equation of state [6]. For the particle part, the transport equation for the modeled joint PDF of fluctuating velocity, turbulent frequency, and compositions is derived from the modeled joint velocityturbulent frequency-compositions PDF transport equation and is solved by a particle-based Monte Carlo method. The FV and particle algorithm are then coupled by exchanging the mean fields required to close the mean conservation and particle evolution equations. The FV and particle system of equations are briefly described in the following sections and the readers are referred to the references $[5,6]$ for a more complete description of governing equations.

\subsubsection{FV system}

A FV method is used to solve the mean mass, mean momentum, and mean energy conservation equations coupled with the mean equation of state. The conservation equations for mean mass and mean momentum are directly derived from the modeled PDF transport equation and are given by

$$
\begin{aligned}
& \frac{\partial}{\partial t}\langle\rho\rangle+\frac{\partial}{\partial x_{i}}\left(\langle\rho\rangle \tilde{U}_{i}\right)=0, \\
& \frac{\partial}{\partial t}\left(\langle\rho\rangle \tilde{U}_{i}\right)+\frac{\partial}{\partial x_{j}}\left(\langle\rho\rangle \tilde{U}_{i} \tilde{U}_{j}+\langle p\rangle \delta_{i j}\right) \\
& =-\frac{\partial}{\partial x_{j}}\left(\langle\rho\rangle \widetilde{u_{i} u_{j}}\right) .
\end{aligned}
$$

The primary representation of the thermochemistry is in the particle system. Based on the particle properties, the equivalent sensible internal energy $\epsilon_{s}$ is defined such that

$\epsilon_{s} \equiv \frac{1}{\gamma_{0}-1} \frac{p}{\rho}$

where $\gamma_{0}=1.4$. It is emphasized here that there are no restrictive assumptions implied, e.g., constant specific heats, in this formulation. If the composition is represented as an ideal gas mixture, then $\boldsymbol{\epsilon}_{s}$ is given by

$\epsilon_{s}=\frac{\mathscr{R} T}{\gamma_{0}-1} \sum_{i} Y_{i} / W_{i}$

where $\mathscr{R}$ is the universal gas constant, $T$ is the temperature, and $Y_{i}$ and $W_{i}$ are the mass fraction and molecular weight of species $i$. The mean $\tilde{\epsilon}_{s}$ is transferred from particle system to the FV system.

In the FV system, the energy variable used is

$\tilde{E}_{s}=\tilde{\epsilon}_{s}+\frac{1}{2} \tilde{U}_{i} \tilde{U}_{i}$,

so that the mean equation of state is

$\langle p\rangle=\left(\gamma_{0}-1\right)\langle\rho\rangle\left(\tilde{E}_{s}-\frac{1}{2} \tilde{U}_{i} \tilde{U}_{i}\right)$.

The transport equation solved for $\tilde{E}$ is

$\frac{\partial}{\partial t}\left(\langle\rho\rangle \tilde{E}_{s}\right)+\frac{\partial}{\partial x_{i}}\left(\tilde{U}_{i}\left(\langle\rho\rangle \tilde{E}_{s}+\langle p\rangle\right)\right)=S_{E}$,

where the source term $S_{E}$ is determined by the energy correction algorithm [6] to ensure that the energy $\tilde{\epsilon}_{s}=\tilde{E}_{s}-\frac{1}{2} \tilde{U}_{i} \tilde{U}_{i}$ in the FV system is consistent with that obtained from the particles.

\subsubsection{Particle system}

As mentioned above, a particle-based Monte Carlo method is used to solve the transport equation of the joint PDF of the fluctuating velocity, turbulent frequency, and compositions. In this method, the fluid is represented by a large set of particles having properties that evolve according to the model stochastic differential equations (SDEs) in a way such that the particles exhibit the same PDF as the solution of the modeled joint PDF transport equation. Each particle has the primary properties of mass $\left(m^{*}\right)$, position $\left(\mathbf{X}^{*}\right)$, fluctuating velocity $\left(\mathbf{u}^{*}\right)$, turbulent frequency $\left(\omega^{*}\right)$, and compositions $\left(\boldsymbol{\phi}^{*}\right)$, and the secondary properties which are either derived from the primary properties such as temperature and Reynolds stresses or interpolated from the FV mean fields such as mean velocity $\left(\tilde{\mathbf{U}}^{*}\right)$ and mean pressure $\left(\langle p\rangle^{*}\right)$. Note that the superscript “*” denotes that the quantity is a particle property.

The increment $d \mathbf{X}^{*}$ in particle position over an infinitesimal time interval $d t$ is given by

$d \mathbf{X}^{*}=\left(\tilde{\mathbf{U}}^{*}+\mathbf{u}^{*}\right) d t$.

The fluctuating velocity $\mathbf{u}^{*}$ evolves according to the simplified Langevin model (SLM) [4]

$$
\begin{aligned}
d u_{i}^{*}(t)= & \frac{1}{\langle\rho\rangle} \frac{\partial\left(\langle\rho\rangle \widetilde{u_{i} u_{j}}\right)}{\partial x_{j}} d t-u_{j}^{*} \frac{\partial \tilde{U}_{i}}{\partial x_{j}} d t \\
& -\left(\frac{1}{2}+\frac{3}{4} C_{0}\right) \Omega u_{i}^{*}(t) d t+\left(C_{0} k \Omega\right)^{1 / 2} d W_{i},
\end{aligned}
$$

where $k=\frac{1}{2} \widetilde{u_{i} u_{i}}$ is the mean turbulent kinetic energy, $C_{0}$ is a model constant (Table 1), $d W_{i}$ is an isotropic Wiener process and $\Omega$ is the conditional mean turbulent frequency defined as 
Table 1

Model constants

\begin{tabular}{lll}
\hline Constant & $\begin{array}{l}\text { Standard } \\
\text { value }\end{array}$ & Used in \\
\hline$C_{0}$ & 2.1 & SLM \\
$C_{\Omega}$ & 0.6893 & definition of $\Omega$ \\
$C_{\omega 1}$ & 0.65 & turbulence frequency model \\
$C_{\omega 2}$ & 0.9 & turbulence frequency model \\
$C_{3}$ & 1.0 & turbulence frequency model \\
$C_{4}$ & 0.25 & turbulence frequency model \\
$C_{\phi}$ & 2.0 & IEM mixing model \\
\hline
\end{tabular}

$\Omega \equiv C_{\Omega} \frac{\left\langle\rho^{*} \omega^{*} \mid \omega^{*} \geq \tilde{\omega}\right\rangle}{\langle\rho\rangle}$,

where $C_{\Omega}$ is another model constant (Table 1). The conditioning in Eq. 12 excludes the particles with turbulent frequency less than the mean turbulent frequency, that is, $\omega^{*}<\tilde{\omega}$, in evaluating the conditional mean turbulent frequency $\Omega$ to approximate the assumption that the rate of turbulent processes in intermittent regions is represented by the turbulent particles better than the average of turbulent and nonturbulent particles [8].

The turbulent frequency $\omega^{*}$ provides a time scale to close Eqs. 12 and 13. In the present PDF method, the turbulent frequency is represented as a particle property and it evolves by the stochastic model [8] given by

$$
\begin{aligned}
d \omega^{*}(t)= & -C_{3}\left(\omega^{*}-\tilde{\omega}\right) \Omega d t-S_{\omega} \Omega \omega^{*}(t) d t \\
& +\left(2 C_{3} C_{4} \tilde{\omega} \Omega \omega^{*}(t)\right)^{1 / 2} d W,
\end{aligned}
$$

where $W$ is an independent Wiener process, and the source term $S_{\omega}$ is defined as

$S_{\omega}=C_{\omega 2}-C_{\omega 1} \frac{P}{k \Omega}$

where $P=-\widetilde{u_{i} u_{j}}\left(\partial \tilde{U}_{i} / \partial x_{j}\right)$ is the turbulent production. The model constants $C_{\omega 1}, C_{\omega 2}, C_{3}$, and $C_{4}$ are specified in Table 1.

The chemical reactions are treated by a simple flamelet model. The flamelet chemistry model views a turbulent flame as an ensemble of thin quasi-laminar flame zones called flamelets and relies on the assumption that the chemical time scales are much shorter than the convective and diffusion time scales. The flamelet model relaxes the infinitely fast chemistry assumption used in the flame sheet model by utilizing the scalar dissipation rate as a parameter to describe the degree of departure from the equilibrium. Under the condition of widely separated time scales, Peters [20] showed that in the steady state, the thermochemical properties can be related to the mix- ture fraction and scalar dissipation rate, and the flamelet libraries can be formed by solving flamelet model equations for a series of scalar dissipation rates. An extensive review of the flamelet model can be found in [20]. In the present study, the flamelet approach is further simplified and the flamelet library is formed based on the laminar flame calculations at a moderate stretch rate (namely $a=100 \mathrm{1} / \mathrm{s}$ ) using the GRI 2.1 detailed chemistry model [21]. With this simplification, the flamelet model reduces to be a look up table and the thermochemical state of the particle is characterized solely by the mixture fraction. Following Bilger [22], the mixture fraction is defined as [11]

$\xi=$

$$
\frac{\frac{2\left(Z_{C}-Z_{C, O}\right)}{W_{C}}+\frac{\left(Z_{H}-Z_{H, O}\right)}{2 W_{H}}-\frac{2\left(Z_{O}-Z_{O, O}\right)}{W_{O}}}{\frac{2\left(Z_{C, F}-Z_{C, O}\right)}{W_{C}}+\frac{\left(Z_{H, F}-Z_{H, O}\right)}{2 W_{H}}-\frac{2\left(Z_{O, F}-Z_{O, O}\right)}{W_{O}}},
$$

where $Z_{i}$ is a conserved scalar given by the total mass fraction of element $i$ and $W_{i}$ is the molecular weight of element $i$. The first subscripts $C, H$, and $O$ denote carbon, hydrogen and oxygen, respectively. The second subscripts $F$ and $O$ refer to the fuel and air streams, respectively. In this simple chemistry model, it is sufficient to represent only $\xi$ explicitly, and all the other thermochemical variables can be obtained from the flamelet properties (as function of $\xi)$.

Finally the effects of molecular mixing are accounted for by the interaction by exchange with the mean (IEM) mixing model [23]. For the present simple flamelet chemistry model, the IEM mixing model can be given by

$d \xi^{*}=-\frac{1}{2} C_{\phi} \Omega\left(\xi^{*}-\tilde{\xi}^{*}\right) d t$,

where the model constant $C_{\phi}$ is specified in Table 1 . According to the IEM model, the particles composition relaxes to the mean composition in the composition space at the rate determined by the inverse of the turbulent time scale $\tau^{-1}=\Omega$.

The validity of the flamelet chemistry model and the IEM mixing model is questionable especially downstream of the recirculation zones where significant local extinction and reignition occur [11]. However, considering the scope of the present study, the use of the flamelet model in conjunction with the IEM mixing model is justified here as it facilitates extensive numerical experiment. 

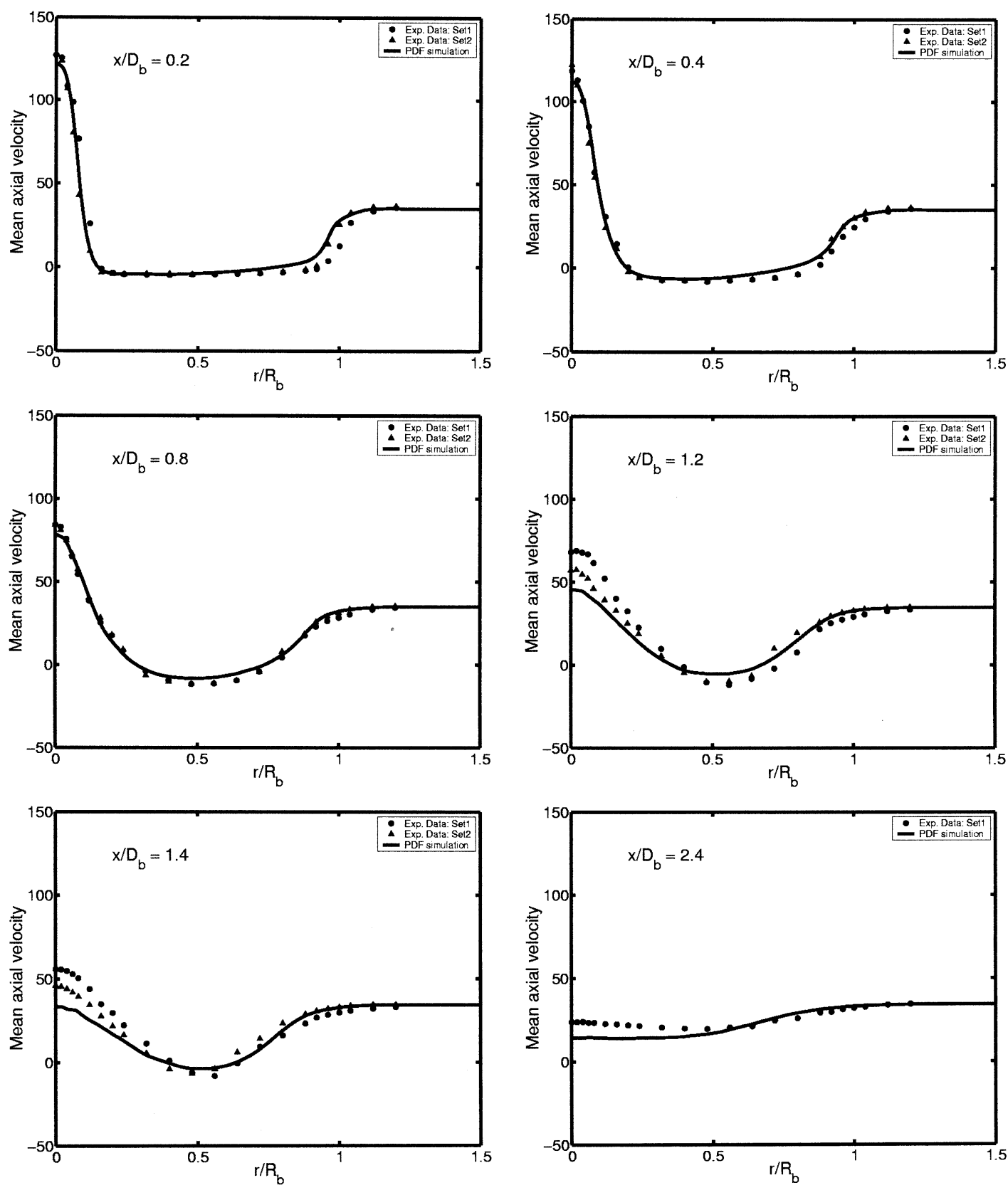

Fig. 1. Mean axial velocity $(\tilde{U}(\mathrm{~m} / \mathrm{s}))$ profiles at the axial locations $0.2 D_{b}, 0.4 D_{b}, 0.8 D_{b}, 1.2 D_{b}, 1.4 D_{b}$, and $2.4 D_{b}$. Symbols denote the experimental data and solid lines denote the PDF simulations. Grid: $176 \times 136, N_{p c}=50, N_{T A}^{P 2 P}=500$.

\subsection{Numerical solution algorithm}

The consistent hybrid FV/particle method has proved to be an efficient way of solving the modeled PDF transport equation $[5,6]$. The basic idea in the hybrid approach is that a FV method is used to solve the mean conservation equations for mass, momentum and energy coupled with the mean equation of state (Eqs. 3, 4, 8, and 9) and a particle-based Monte Carlo method is used to solve the modeled PDF transport equation for the fluctuating velocity, turbulent frequency and compositions (Eqs. 10, 11, 13, and 16).

The FV and particle codes are coupled to form a complete solution algorithm as follows. The mean velocity and mean pressure fields are supplied to the 

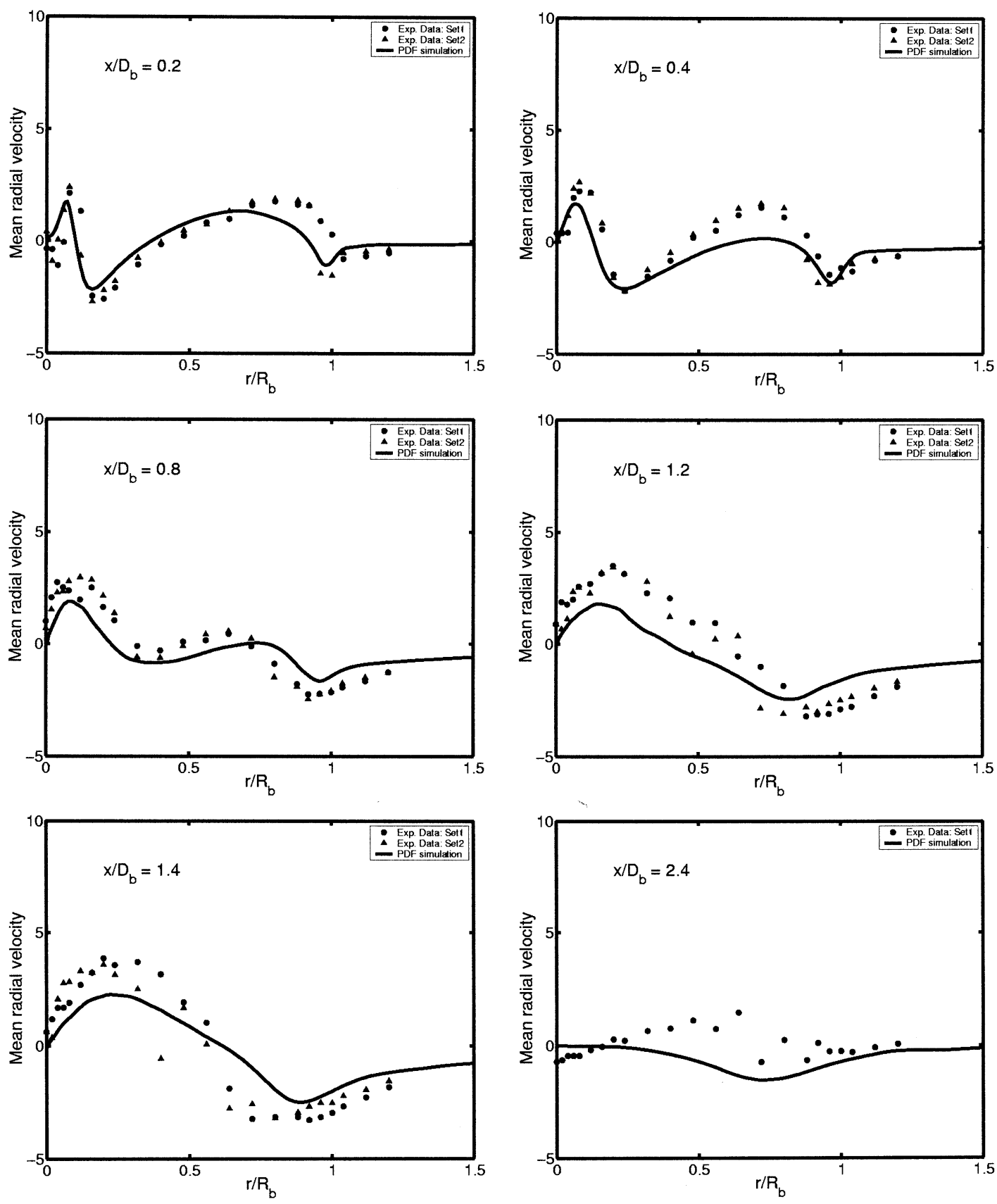

Fig. 2. Mean radial velocity $(\tilde{V}(\mathrm{~m} / \mathrm{s}))$ profiles at the axial locations $0.2 D_{b}, 0.4 D_{b}, 0.8 D_{b}, 1.2 D_{b}, 1.4 D_{b}$, and $2.4 D_{b}$. Symbols denote the experimental data and solid lines denote the PDF simulations. Grid: $176 \times 136, N_{p c}=50, N_{T A}^{P 2 P}=500$.

particle code by the FV code and the FV code together with the energy correction algorithm [6] gets all the Reynolds stresses, the chemical source term and the mean equivalent sensible internal energy fields from the particle code. Note that the noisy scalar fluxes are not included in the mean energy conservation equation (Eq. 9) since the energy cor- rection algorithm guarantees that the FV mean equivalent sensible internal energy relaxes to its particle counterpart no matter which terms are included in the energy source term as far as a steady state is reached [6]. It is stressed again here that the present hybrid method is completely consistent at the level of governing equations since, as mentioned earlier all the 

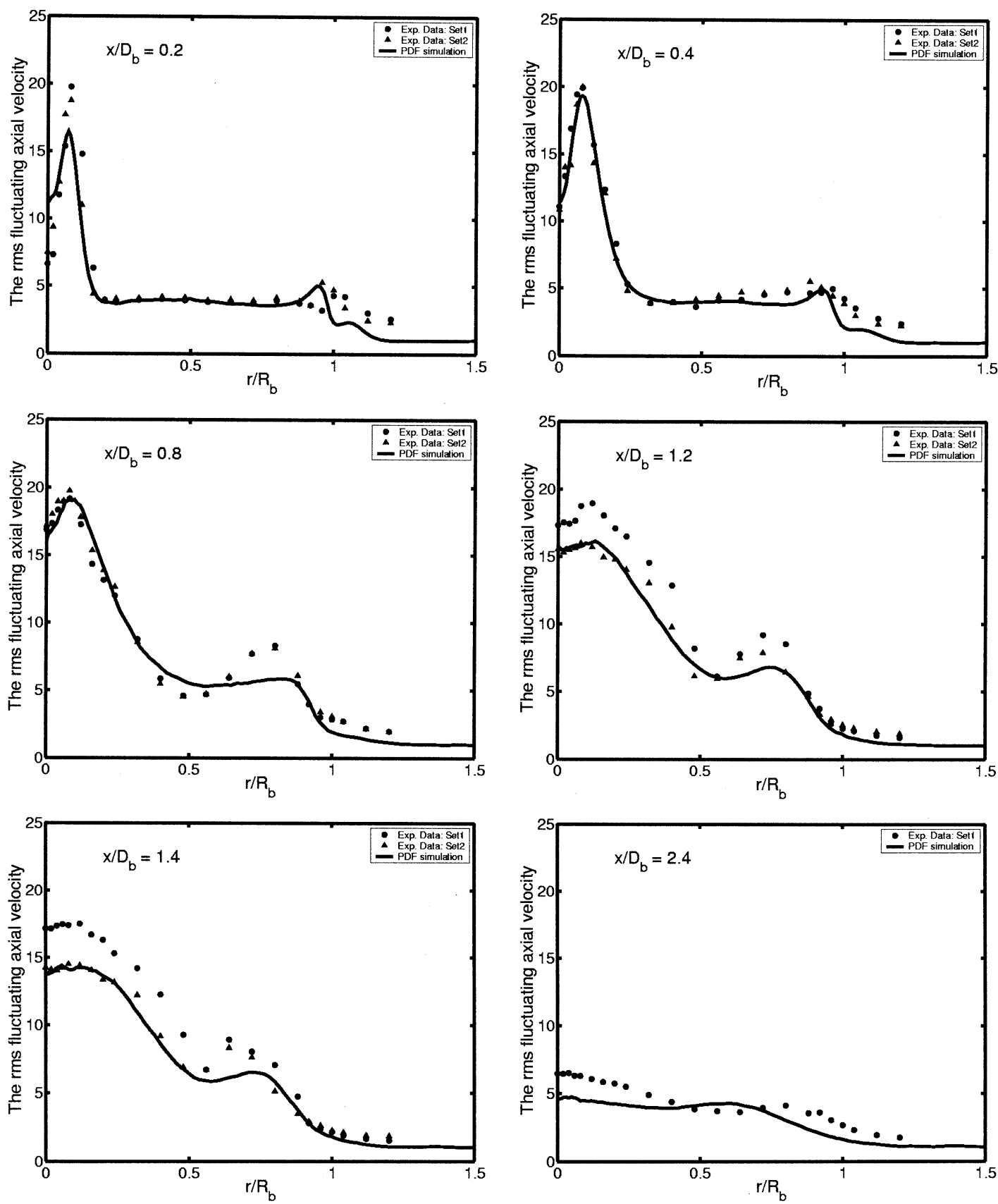

Fig. 3. The rms fluctuating axial velocity $\left(u^{\prime}(\mathrm{m} / \mathrm{s})\right)$ profiles at the axial locations $0.2 D_{b}, 0.4 D_{b}, 0.8 D_{b}, 1.2 D_{b}, 1.4 D_{b}$, and $2.4 D_{b}$. Symbols denote the experimental data and solid lines denote the PDF simulations. Grid: $176 \times 136, N_{p c}=50, N_{T A}^{P 2 P}$ $=500$.

equations solved by the FV and particle algorithms are directly derived from the modeled PDF transport equation. Furthermore a full consistency at the numerical solution level is enforced by the correction algorithms [6].

The form of coupling between the FV and particle algorithm is of crucial importance and distinguishes different types of hybrid method. There exist infinite number of coupling strategies between the two extreme cases, namely the tight and loose coupling methods. In a tight coupling, the FV and particle codes are run for a single time step to complete an 

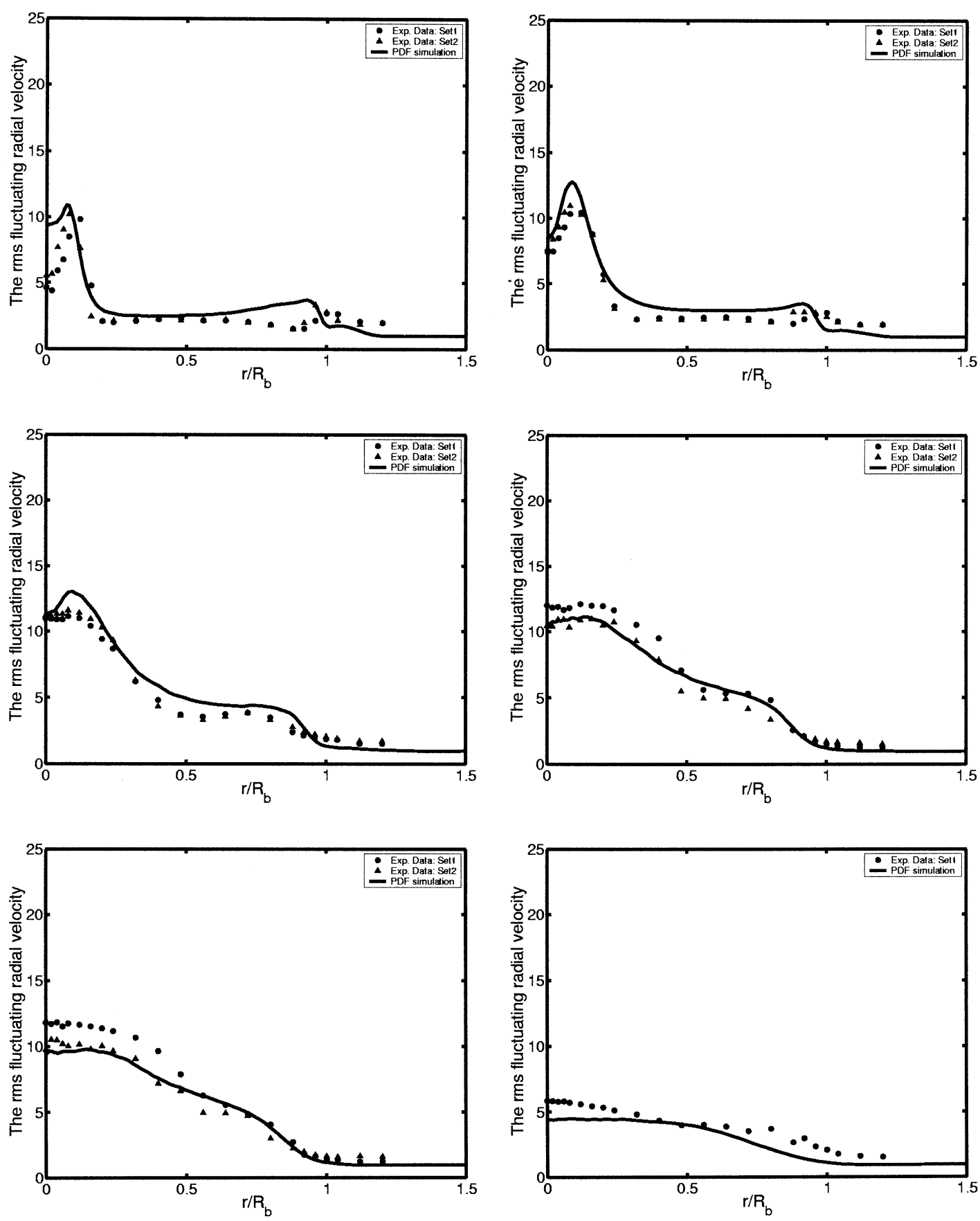

Fig. 4. The rms fluctuating radial velocity $\left(v^{\prime}(\mathrm{m} / \mathrm{s})\right)$ profiles at the axial locations $0.2 D_{b}, 0.4 D_{b}, 0.8 D_{b}, 1.2 D_{b}, 1.4 D_{b}$, and $2.4 D_{b}$. Symbols denote the experimental data and solid lines denote the PDF simulations. Grid: $176 \times 136, N_{p c}=50, N_{T A}^{P 2 P}$ $=500$.

outer iteration while, in the loosely coupled case, an outer iteration is completed by running the $\mathrm{FV}$ and particle codes separately until reaching a pseudo steady state. Earlier studies [6,24] have shown that neither the tight nor the loose coupling is optimal in terms of numerical efficiency and robustness. The tight coupling seems to improve the robustness of the hybrid method while the more loosely coupled algorithm leads to a better global convergence rate to steady state. Therefore, as a compromise between the robustness and fast convergence of the hybrid algorithm, a pseudo loose coupling in which an outer 

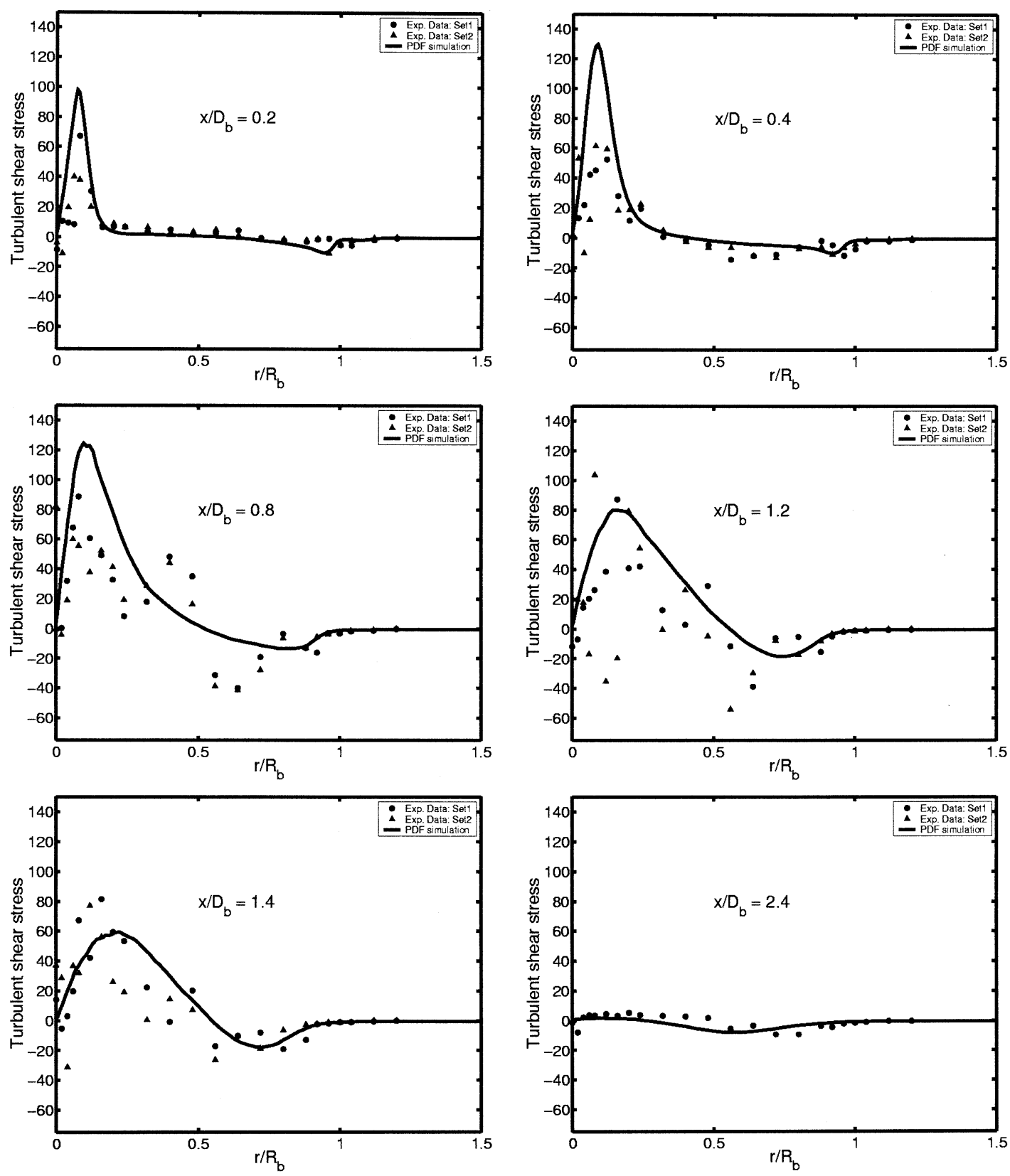

Fig. 5. Mean turbulent shear stress $\left(\widetilde{u v}\left(\mathrm{~m}^{2} / \mathrm{s}^{2}\right)\right)$ profiles at the axial locations $0.2 D_{b}, 0.4 D_{b}, 0.8 D_{b}, 1.2 D_{b}, 1.4 D_{b}$, and $2.4 D_{b}$. Symbols denote the experimental data and solid lines denote the PDF simulations. Grid: $176 \times 136, N_{p c}=50, N_{T A}^{P 2 P}=500$.

iteration is completed by running both the FV and particle codes each for a specified number of time steps has been found to give the best asymptotic global convergence rate without loss of robustness [6]. In the present study, the FV and particle codes are run for 10 and 3 time steps, respectively, in each outer iteration throughout the computations.

Such a pseudo loosely coupled hybrid algorithm has been implemented in the $H Y B 2 D$ code [6]. In this code, the particle algorithm is largely based on the PDF $2 D V$ [25] code and the FV algorithm is a slight modification of the alternating direction implicit (ADI) scheme of Caughey [26]. The local time stepping algorithm that has been recently developed and shown to significantly accelerate the global convergence of the hybrid method [7] is also incorporated into the present hybrid method. Note that a similar local time stepping procedure has been recently presented by Mobus et 

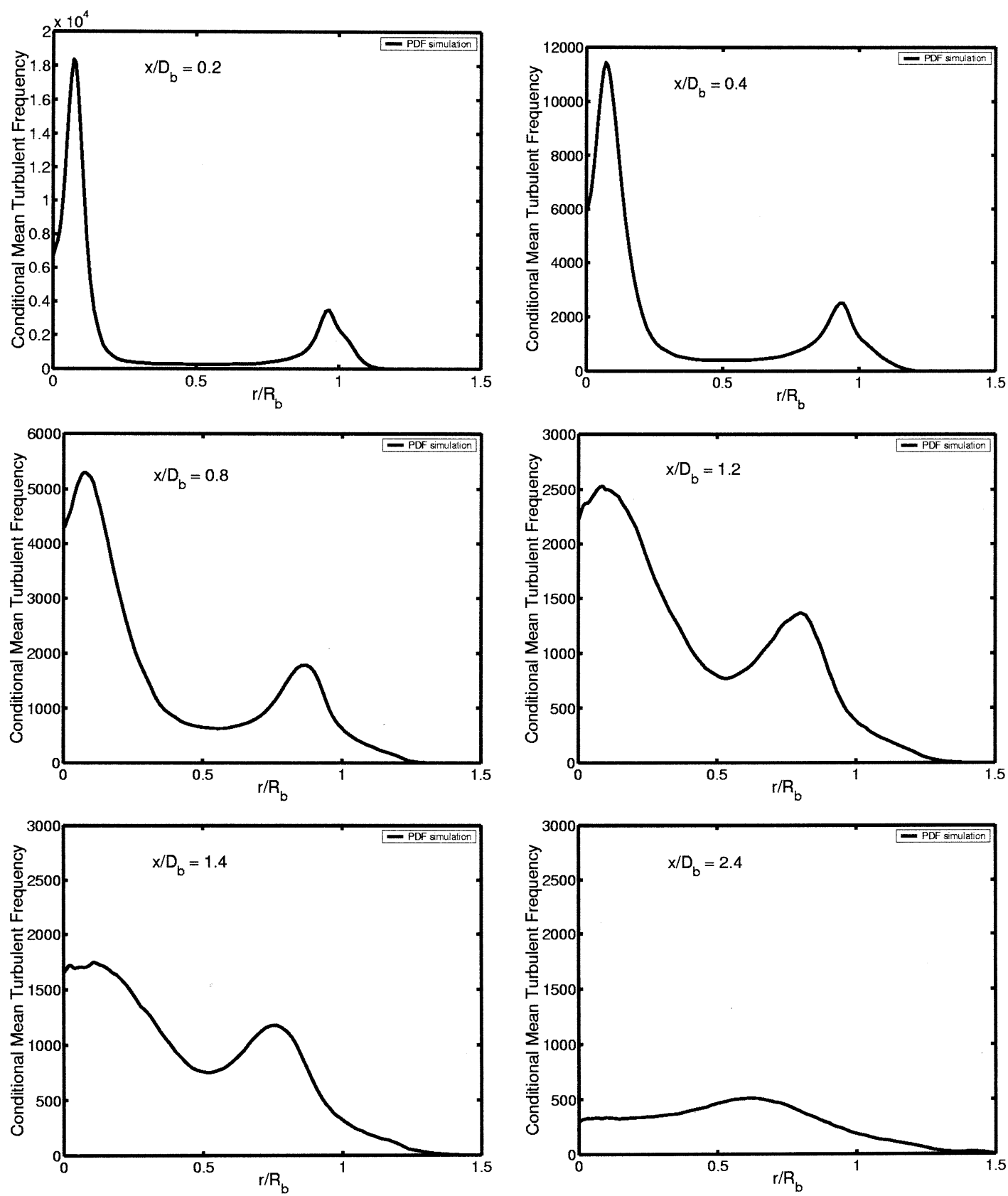

Fig. 6. Conditional mean turbulent frequency $(\Omega(1 / s))$ profiles at the axial locations $0.2 D_{b}, 0.4 D_{b}, 0.8 D_{b}, 1.2 D_{b}, 1.4 D_{b}$, and $2.4 D_{b}$. Solid lines denote the PDF simulations. Grid: $176 \times 136, N_{p c}=50, N_{T A}^{P 2 P}=500$.

al. [27] for convergence acceleration of Lagrangian PDF methods. A complete discussion about the consistent hybrid algorithm and the local time stepping method can be found in [5,6] and [7], respectively.

\subsection{Time-averaging method}

Time-averaging is an essential part of the hybrid solution algorithm and is a very useful tool to reduce the statistical fluctuations in the FV and particle mean fields without increasing the number of particles. In the present study, both the FV and particle fields are time-averaged. Following Muradoglu et al. [6], for a mean field $Q$, time-averaged mean $Q_{T A}$ is evaluated as

$Q_{T A}^{k}=\left(1-\frac{1}{N_{T A}}\right) Q_{T A}^{k-1}+\frac{1}{N_{T A}} Q^{k}$ 

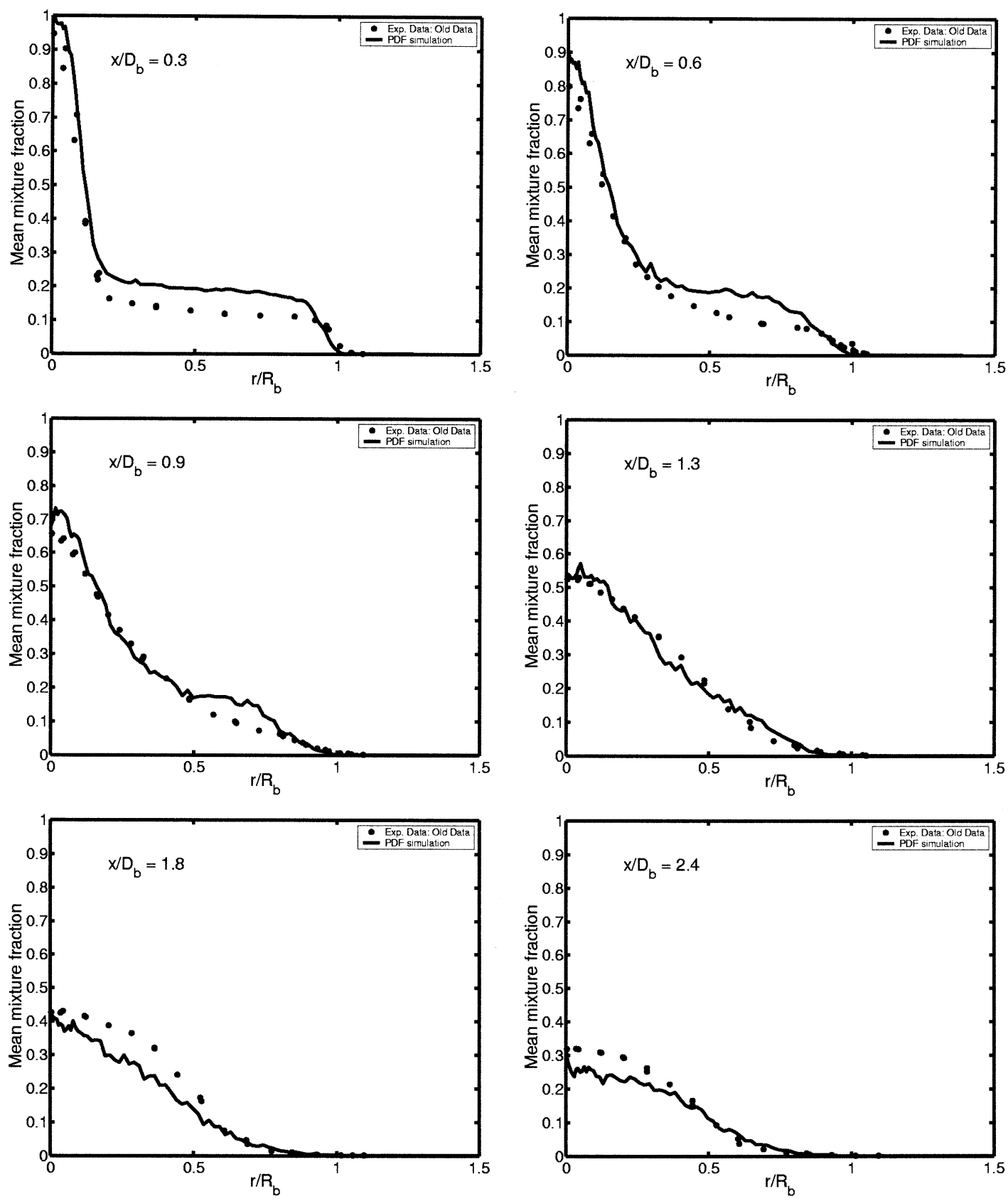

Fig. 7. Mean mixture fraction $(\tilde{\xi})$ profiles at the axial locations $0.3 D_{b}, 0.6 D_{b}, 0.9 D_{b}, 1.3 D_{b}, 1.8 D_{b}$, and $2.4 D_{b}$. Symbols denote the experimental data and solid lines denote the PDF simulations. Grid: $176 \times 136, N_{p c}=50, N_{T A}^{P 2 P}=500$. (see footnote on p. 130.)

where the superscript $k$ denotes the number of particle time steps for the particle fields and the number of outer iteration for the FV fields, and $N_{T A}$ is a time-averaging factor to be specified. In the present study, the time-averaging factor is specified differently for the FV fields, the particle fields used in the mean conservation equations solved by the FV algorithm and the particle fields used in the particle evolution equations, and are denoted by $N_{T A}^{F V}, N_{T A}^{P 2 F V}$, and $N_{T A}^{P 2 P}$, respectively. In all the results presented here, the parameters $N_{T A}^{F V}$ and $N_{T A}^{P 2 F V}$ are fixed to the values 5 and 10 , respectively, throughout the computations while the parameter $N_{T A}^{P 2 P}$ is set to 20 initially and is kept at this value 

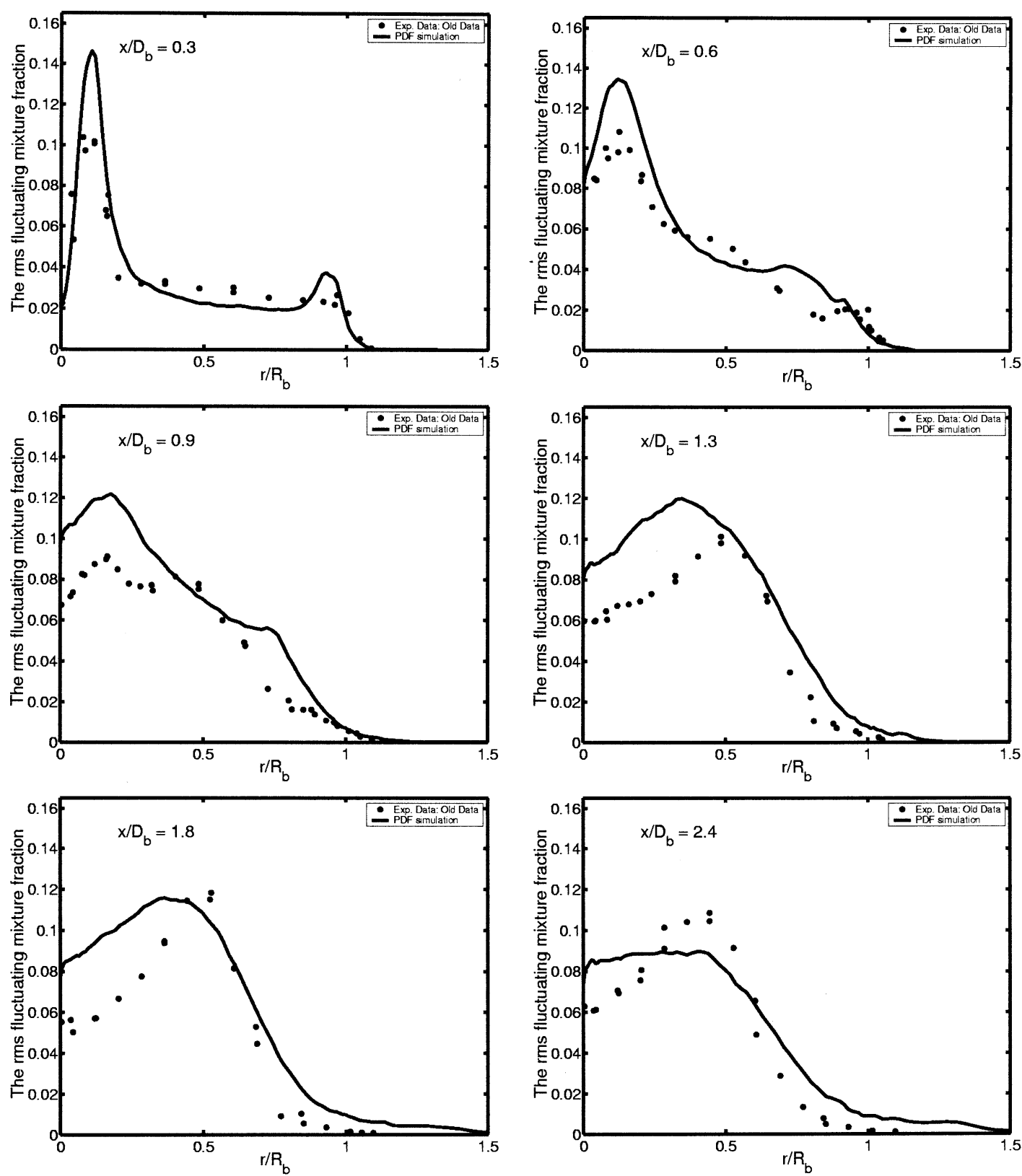

Fig. 8. The rms fluctuating mixture fraction $\left(\xi^{\prime \prime}\right)$ profiles at the axial locations $0.3 D_{b}, 0.6 D_{b}, 0.9 D_{b}, 1.3 D_{b}, 1.8 D_{b}$, and $2.4 D_{b}$. Symbols denote the experimental data and solid lines denote the PDF simulations. Grid: $176 \times 136, N_{p c}=50, N_{T A}^{P 2 P}=500$. (see footnote on p. 130.)

until a statistically stationary state is approached and then it is gradually increased to its final value. Note that the selection of the initial value and the starting time for setting $N_{T A}^{P 2 P}$ to approach its final value are totally arbitrary and have no effects on the accuracy of the numerical solution as long as a statistically steady state is reached. From now on $N_{T A}^{P 2 P}$ denotes the final value of this time-averaging factor.

\section{Results and discussion}

\subsection{Flow and boundary conditions}

The velocity-turbulent frequency-compositions PDF method combined with the consistent hybrid algorithm is applied to a bluff-body stabilized nonpremixed turbulent flame. The bluff body stabilized 

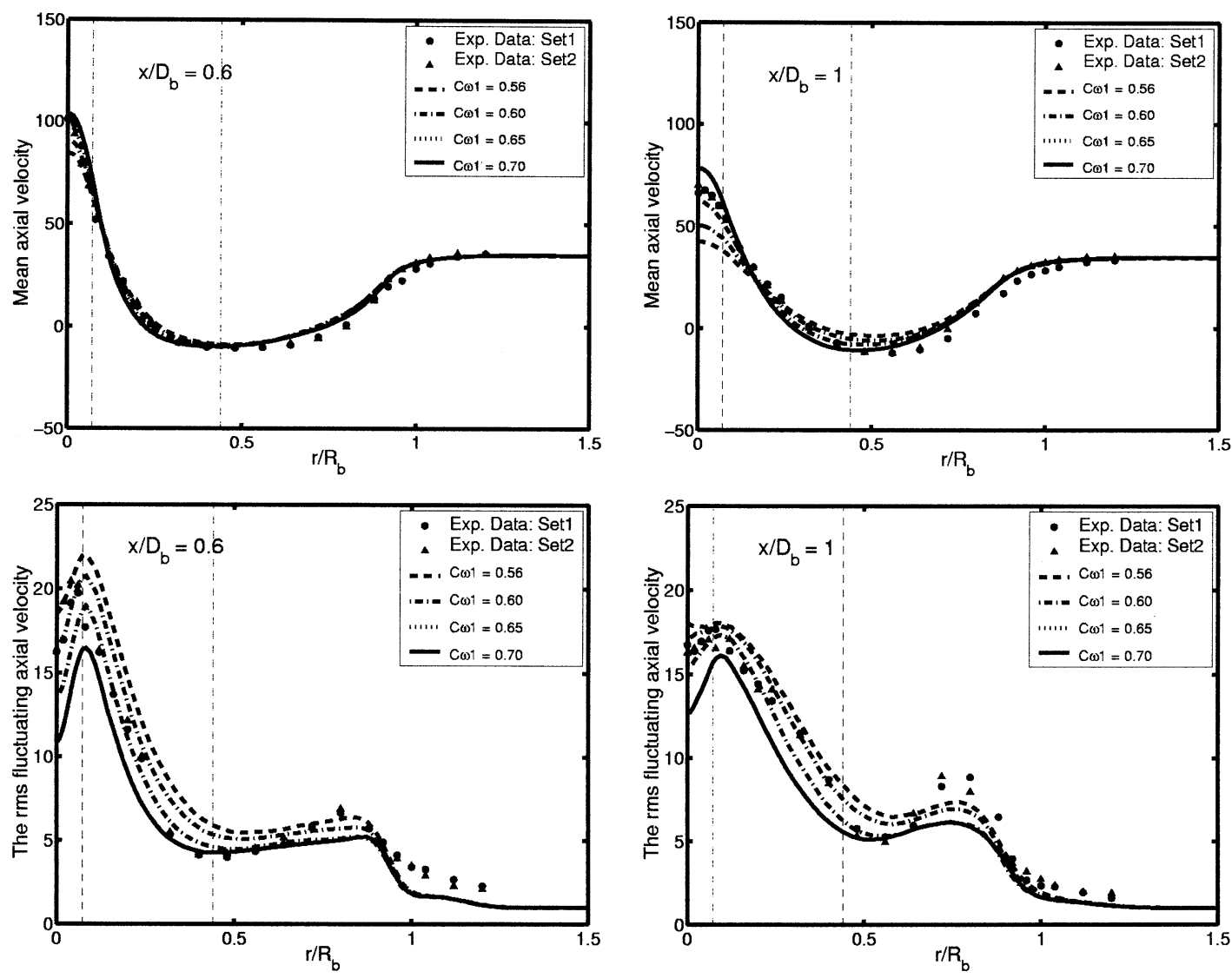

Fig. 9. Profiles of mean axial velocity $(\tilde{U}(\mathrm{~m} / \mathrm{s}))$ (top plots) and the rms fluctuating axial velocity $\left(u^{\prime}(\mathrm{m} / \mathrm{s})\right)$ (bottom plots) at the axial locations $0.6 D_{b}$ (left plots) and $D_{b}$ (right plots) computed on $128 \times 96$ grid and $C_{\omega 1}$ ranges from 0.56 to 0.7. $N_{T A}^{P 2 P}$ $=500$.

turbulent flames have received significant attention in recent years and been studied experimentally and numerically $[9,10,15-19,28,29]$.

Besides their practical relevance, the bluff-body flames are challenging test cases for turbulence and chemistry models as well as for numerical solution algorithms. The bluff body flame investigated here is a statistically axisymmetric, non-premixed turbulent flame consisting of a central fuel jet $\left(50 \% \mathrm{H}_{2}\right.$ and $50 \% \mathrm{CH}_{4}$ by volume) with diameter $2 R_{j}=3.6 \mathrm{~mm}$, a bluff body surrounding the fuel jet with diameter $D_{b}=50 \mathrm{~mm}$ and an unconfined coflowing stream of air surrounding the bluff-body. The flame is stabilized behind the bluff body by the intense mixing of fuel and air and by the hot products providing a continuous ignition source in the recirculation zone. This bluff body stabilized flame was first studied experimentally by Dally et al. [9] in which the jet and coflow bulk velocities were chosen as $118 \mathrm{~m} / \mathrm{s}$ and 40 $\mathrm{m} / \mathrm{s}$, respectively, and the measurements were taken both for the flow and scalar fields. These experimental results are labeled here as "old data." The flow field measurements for the same flame but with slightly reduced jet and coflow bulk velocities of 108 $\mathrm{m} / \mathrm{s}$ and $35 \mathrm{~m} / \mathrm{s}$, respectively, have been recently repeated twice by Kalt and Masri [10], and these new sets of data are labeled here as "set1" and "set2." In the present study, essentially the new sets of data are used for comparisons with the PDF calculations and the old data are used only for the mean mixture fraction and the rms fluctuating mixture fraction fields since no scalar field measurements have been provided for the new case of the reduced bulk velocities. In both experiments, the flow fields were measured by using a Laser Doppler Velocimetry (LDV) technique and the scalar fields were measured by using a single-point Raman-Rayleigh Laser Induced Fluorescence (LIF) method. A complete description of the experimental set up and the experimental data are available on Internet [29].

For the numerical simulations, a cylindrical coordinate system is adopted with $x$ representing the axial direction aligned with the jet axis and $r$ the radial direction. The origin of the coordinate system is 

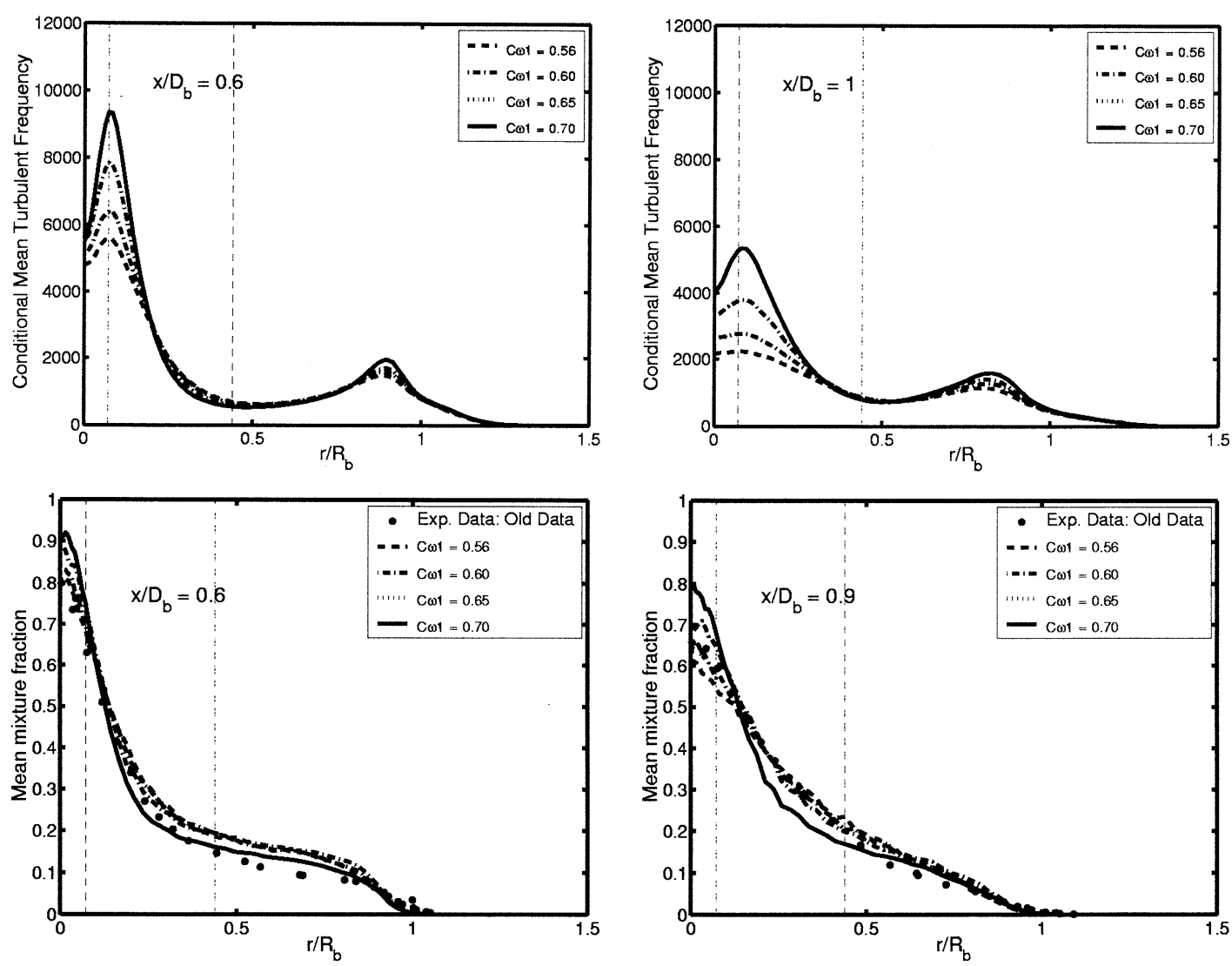

Fig. 10. Profiles of conditional mean turbulent frequency $(\Omega(1 / s))$ (top plots) and mean mixture fraction $(\tilde{\xi})$ (bottom plots) at the axial locations $0.6 D_{b}$ (left plots), and $D_{b}$ for $\Omega$ and $0.9 D_{b}$ for $\tilde{\xi}$ (right plots) computed on $128 \times 96$ grid and $C_{\omega 1}$ ranges from 0.56 to $0.7 . N_{T A}^{P 2 P}=500$.

placed at the center of the fuel jet in the exit plane. The computational domain is $15 D_{b}$ long in the axial direction and extends to $3 D_{b}$ in the radial direction. A tensor product, orthogonal Cartesian grid is used with the total of $M^{2}$ non-uniform cells. The grid is stretched both in the axial and radial directions.

The initial and boundary conditions are specified in the same way as for the cold bluff-body case studied by Jenny et al. [2] for all the quantities except for that mixture fraction is also specified in the present reacting case at the inlet. The pressure is extrapolated from the computational domain at the inlet boundary. The axial mean velocity is calculated based on the assumption of a fully developed pipe flow in the jet region while it is interpolated from the experimental data in the coflow region. The axial and radial rms fluctuating velocities $\left(u^{\prime}\right.$ and $\left.v^{\prime}\right)$ are also interpolated from the experimental data both in the jet and coflow regions, and the mean turbulent shear stress is then calculated as

$\widetilde{u v}=\rho_{12}\left(u^{\prime 2} v^{\prime 2}\right)^{1 / 2}$, with $\rho_{12}=-0.4$ and $\rho_{12}=0.5\left(r / R_{j}\right)$ in the coflow and jet regions, respectively. Then the particle fluctuating velocity components are specified at the inlet such that the fluctuating velocity PDF is joint normal with zero means. Based on the assumption of equilibrium between the production and dissipation, the mean turbulent frequency is calculated as

$\tilde{\omega}=\frac{P}{k}=-\frac{\widetilde{u v}}{k} \frac{\partial \tilde{U}}{\partial r}$,

where $k$ is the turbulent kinetic energy and $P$ is the mean turbulent energy production rate. Based on the assumption that the flow is dominated by the large recirculation zones and hence there is no need to resolve the boundary layer, a perfect slip, and no penetration boundary conditions are applied on the buff-body surface. At the outflow boundary, the pressure is fixed to the pressure calculated as

$\langle\rho\rangle=\rho_{0}-\langle\rho\rangle \widetilde{v v}$, 

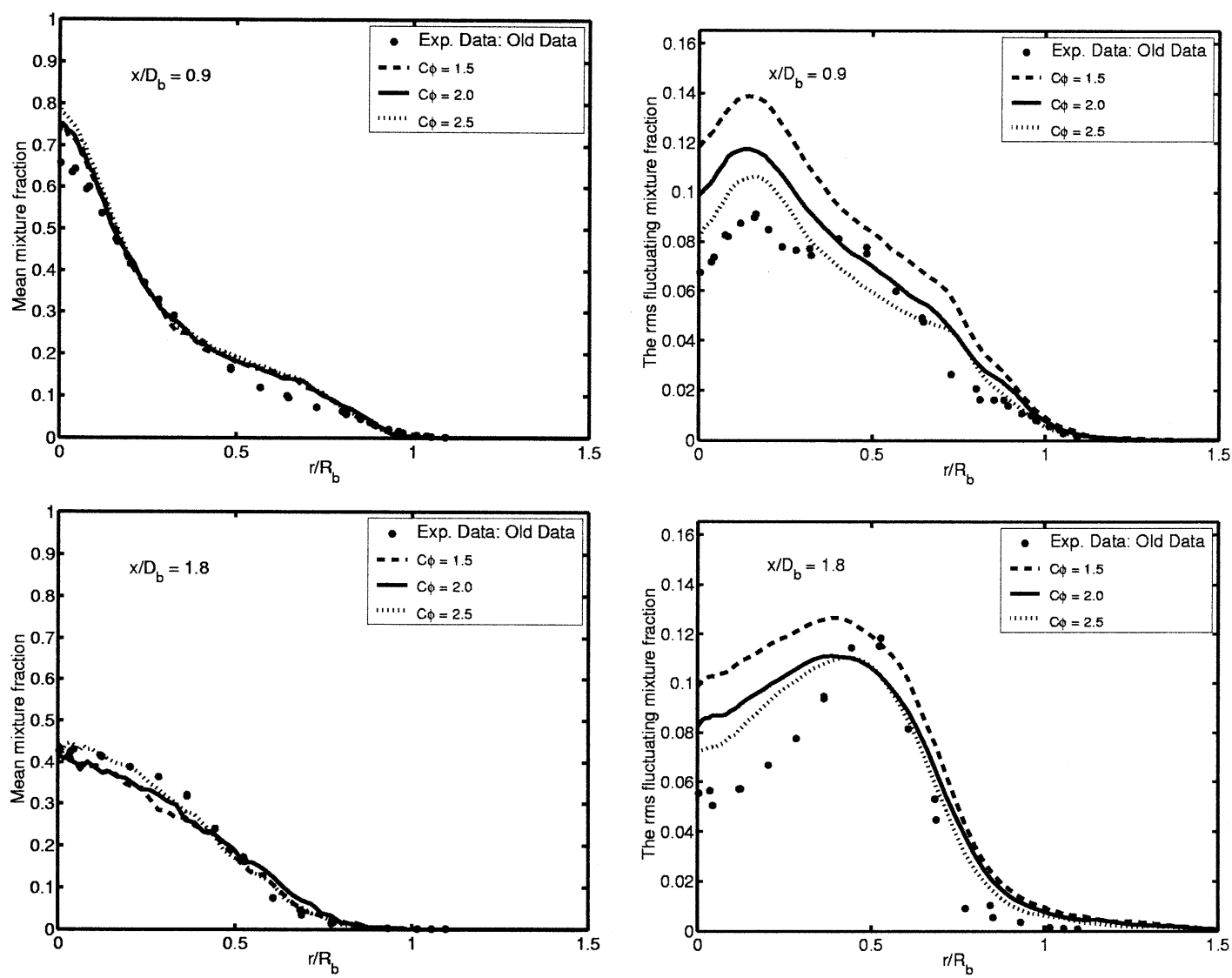

Fig. 11. Profiles of mean mixture fraction $(\tilde{\xi})$ (left plots) and the rms fluctuating mixture fraction ( $\left.\xi^{\prime \prime}\right)$ (right plots) at the axial locations $x=0.9 D_{B}$ (top plots) and $x=1.8 D_{b}$ (bottom plots) with $128 \times 96$ grid and $C_{\phi}$ ranges from 1.5 to 2.5 . $N_{T A}^{P 2 P}=$ 500 .

where $p_{0}$ is the atmospheric pressure $\left(p_{0}=10^{5} \mathrm{~Pa}\right)$ and $v$ is the fluctuating velocity in the radial direction evaluated at the outflow boundary, and all the other quantities are extrapolated from the computational domain. Note that Eq. 20 is exact for plane flows but is only approximately correct for axisymmetric flows [30].

The inlet boundary conditions are also used as the initial conditions.

\subsection{Comparison with experimental data}

To demonstrate the performance of the PDF method combined with the consistent hybrid algorithm, the calculated mean fields are compared with the available experimental data. Note that all the results presented in this section are numerically accurate within 5\% error tolerance as will be shown in section 3.4. Radial profiles of mean axial velocity $(\tilde{U})$, mean radial velocity $(\tilde{V})$, the rms axial fluctuating velocity $\left(u^{\prime}\right)$, the rms radial fluctuating velocity $\left(v^{\prime}\right)$, the mean turbulent shear stress $(\widetilde{u v)}$ and the conditional mean turbulent frequency $(\Omega)$ are plotted in Figs. 1 through 6, respectively, at the axial locations of $x / D_{b}=0.2,0.4,0.8,1.2,1.4$, and 2.4. The conditional mean turbulent frequency is plotted here just to show the overall variations of this quantity because there is no experimental data available to compare with. Fig. 1 shows a remarkably good agreement between the calculated and experimental mean axial velocity profiles up to $x / D_{b}=0.8$ but the mean axial velocity is underpredicted near the centerline in the further downstream locations. However, it is interesting to observe that there are big discrepancies between the two sets of experimental data indicating large uncertainties in the measurements in the far downstream locations where the mean axial velocity is underpredicted. Considering the difficulty to measure and to calculate the mean radial velocity due to its relatively small magnitude compared to the fluctuating component, the calculated mean radial velocity profiles match very well with the experimental 

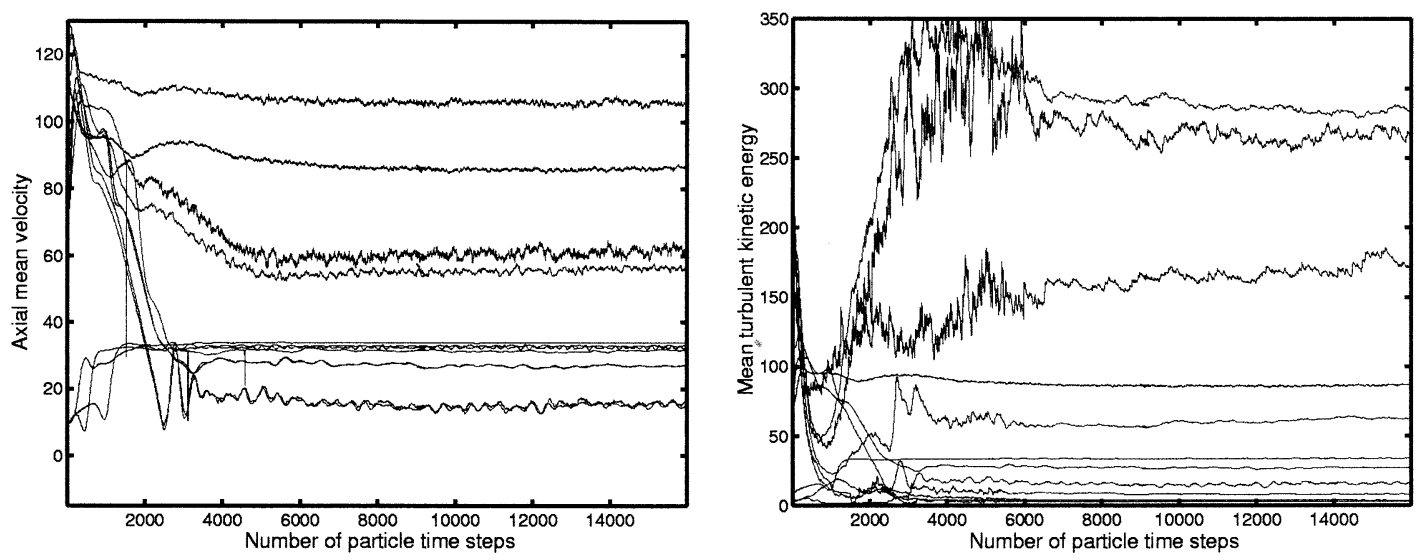

Fig. 12. Convergence histories of mean axial velocity $(\tilde{U}(\mathrm{~m} / \mathrm{s}))$ (left plot) and $\left(k\left(\mathrm{~m}^{2} / \mathrm{s}^{2}\right)\right)$ mean turbulent kinetic energy (right plot). Grid: $176 \times 136, N_{p c}=50, N_{T A}^{P 2 P}=500$.

data at all the locations as shown in Fig. 2. Figs. 1 and 2 together demonstrate that the essential features of the mean flow field are captured well by the present PDF method. As can be seen from Figs. 3 and 4, the rms fluctuating velocities $u^{\prime}$ and $v^{\prime}$ are also well predicted by the PDF method within the recirculation zone, i.e., up to $x / D_{b}=1.2$ but are slightly underpredicted in the further downstream assuming that the true experimental data are simple means of the two sets of measurements. It is interesting to observe that, similar to the mean flow field measurements, there are also large discrepancies between the two sets of experimental data for $u^{\prime}$ and $v^{\prime}$ in the downstream locations of the recirculation zone where these quantities are underpredicted. For the turbulent shear stress (Fig. 5), the experimental data involve up to $30 \%$ error [29] so that it is difficult to make any solid conclusions for this quantity. However, Fig. 5 indicates at least qualitatively good agreement between the PDF results and the experimental data suggesting that the turbulent shear stress field is also well predicted.

The mean mixture fraction $(\tilde{\xi})$ and the rms fluctuations of mixture fraction $\left(\xi^{\prime \prime}\right)$ are plotted in Figs. 7 and 8 , respectively, at the axial locations of $x / D_{b}=0.3,0.6$, $0.9,1.3,1.8$, and 2.4 , and are compared with the available experimental data. ${ }^{\dagger}$ Note that, as mentioned earlier, the experimental data and the calculated results are obtained for the "old case," i.e., with the jet and coflow bulk velocities $118 \mathrm{~m} / \mathrm{s}$ and $40 \mathrm{~m} / \mathrm{s}$, respectively. Fig. 7 demonstrates an overall good agreement between the measured and calculated mean mixture fraction profiles except for the overshoot near the inlet plane behind the bluff-body and undershoot in the far downstream loca-

${ }^{\dagger}$ In Figs. 7 and 8, the first axial location for the calculations is $x / D_{b}=0.3$, whereas the data shown are from $x / D_{b}=0.26$. tions, i.e., $x / D_{b} \geq 1.8$. The rms fluctuations of mixture fraction is overpredicted near the centerline especially in the axial locations of $x / D b \geq 0.9$ as shown in Fig. 8 .

To summarize, considering the simplest turbulence, chemistry, and mixing models are used in the calculations, the mean velocity and scalar fields are overall predicted very well demonstrating the performance of the PDF method combined with the hybrid solution algorithm for this challenging test case.

\subsection{Sensitivity to model constants}

As discussed in Section 2, the present PDF model consists of SLM, JPM, and IEM submodels and earlier studies $[2,12]$ have shown that the PDF simulations are especially sensitive to the model constants $C_{\omega 1}$ in JPM model and $C_{\phi}$ in IEM model. It is not

Table 2

Observation locations used to monitor convergence histories of $\tilde{U}$ and $k ; D_{b}=50 \mathrm{~mm}$ is bluff-body diameter and $R_{j}=1.8 \mathrm{~mm}$ is jet radius

\begin{tabular}{lll}
\hline & $\begin{array}{l}\text { Axial } \\
\text { distance }(x)\end{array}$ & Radial distance $(r)$ \\
\hline Location 1 & $D_{b} / 2$ & 0.0 \\
Location 2 & $D_{b} / 2$ & mid bluff-body $=13.4 \mathrm{~mm}$ \\
Location 3 & $D_{b} / 2$ & $D_{b} / 2$ \\
Location 4 & $D_{b}$ & 0.0 \\
Location 5 & $D_{b}$ & mid bluff-body $=13.4 \mathrm{~mm}$ \\
Location 6 & $D_{b}$ & $D_{b} / 2$ \\
Location 7 & $2 D_{b}$ & 0.0 \\
Location 8 & $2 D_{b}$ & mid bluff-body $=13.4 \mathrm{~mm}$ \\
Location 9 & $2 D_{b}$ & $D_{b} / 2$ \\
Location 10 & $10 D_{b}$ & 0.0 \\
Location 11 & $10 D_{b}$ & mid bluff-body $=13.4 \mathrm{~mm}$ \\
Location 12 & $10 D_{b}$ & $D_{b} / 2$ \\
\hline
\end{tabular}



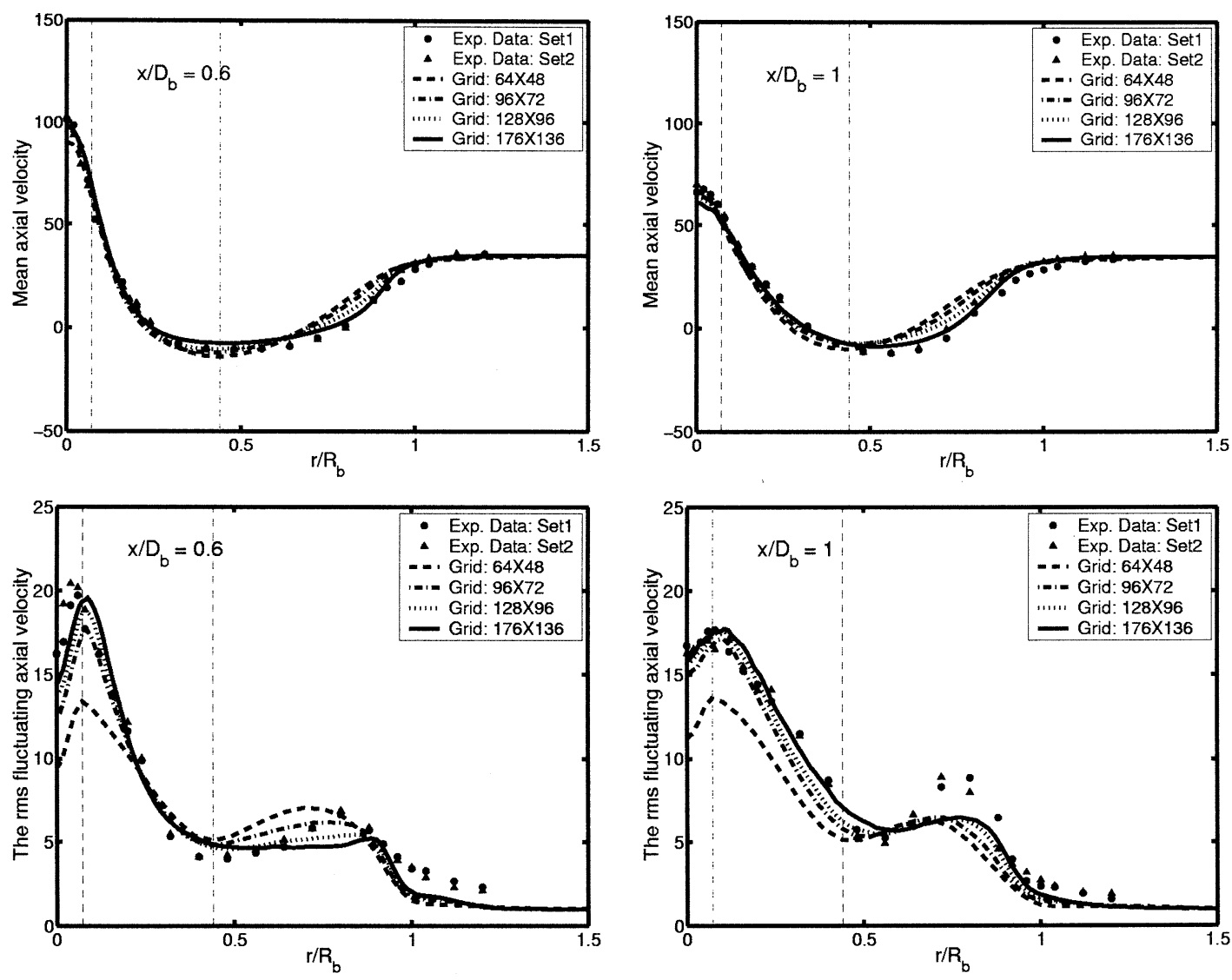

Fig. 13. Profiles of mean axial velocity $(\tilde{U}(\mathrm{~m} / \mathrm{s}))$ (top plots) and the rms fluctuating axial velocity $\left(u^{\prime}(\mathrm{m} / \mathrm{s})\right)$ (bottom plots) at the axial locations $0.6 D_{b}$ (left plots) and $D_{b}$ (right plots) computed on $176 \times 136,128 \times 96,96 \times 72$, and $64 \times 48$ grids. $N_{p c}=100, N_{T A}^{P 2 P}=500$.

intended here to do any systematic parametric study for finding optimal sets of model constants but it is rather intended to show the sensitivities of the numerical solutions to the changes in $C_{\omega 1}$ and $C_{\phi}$ model constants. Therefore, $C_{\omega 1}$ and $C_{\phi}$ are varied to investigate their influence on the numerical calculations.

The direct effects of increasing $C_{\omega 1}$ is to increase the source term in the turbulent frequency equation (Eq. 13) resulting in increasing turbulent frequency, which, in turn leads to reduction in turbulence. The radial profiles of $\tilde{U}$ and $u^{\prime}$, and $\Omega$ and $\tilde{\xi}$ are plotted in Figs. 9 and 10, respectively, for various values of $C_{\omega 1}$ ranging from 0.56 to 0.70 to demonstrate influence of $C_{\omega 1}$ on the numerical calculations. The experimental data for these mean fields except for $\Omega$ are also shown for comparison. It can be seen in these figures that the model constant $C_{\omega 1}$ has a strong influence on all the mean fields but especially on $\Omega$ and $u^{\prime}$ as expected, and the value of $C_{\omega 1}=0.65$ appears to be in better agreement with the experi- mental data as being consistent with our earlier studies $[2,6]$.

The direct effects of increasing the mixing model constant $C_{\phi}$ is to increase the mixing, and hence to decrease the mixture fraction variance $\tilde{\xi}^{\prime 2}$. Fig. 11 shows the profiles of the mean mixture fraction $\tilde{\xi}$ and the rms fluctuating mixture fraction $\xi^{\prime \prime}$ calculated with $C_{\phi}=1.5,2.0$ and 2.5. This figure clearly demonstrates that $C_{\phi}$ has a strong influence on the mixture fraction variance, i.e., increasing $C_{\phi}$ results in big reduction in the mixture fraction variance but the mean mixture fraction seems not very sensitive to the change in $C_{\phi}$. Even though not shown here due to space limitations, mean flow quantities such as $\tilde{U}$ and $u^{\prime}$ are also found not to be very sensitive to changes in $C_{\phi}$.

\subsection{Numerical accuracy}

\subsubsection{Statistical stationarity}

The hybrid method is designed to simulate only statistically stationary flows such as the buff-body flow studied here. Therefore, the statistical stationar- 

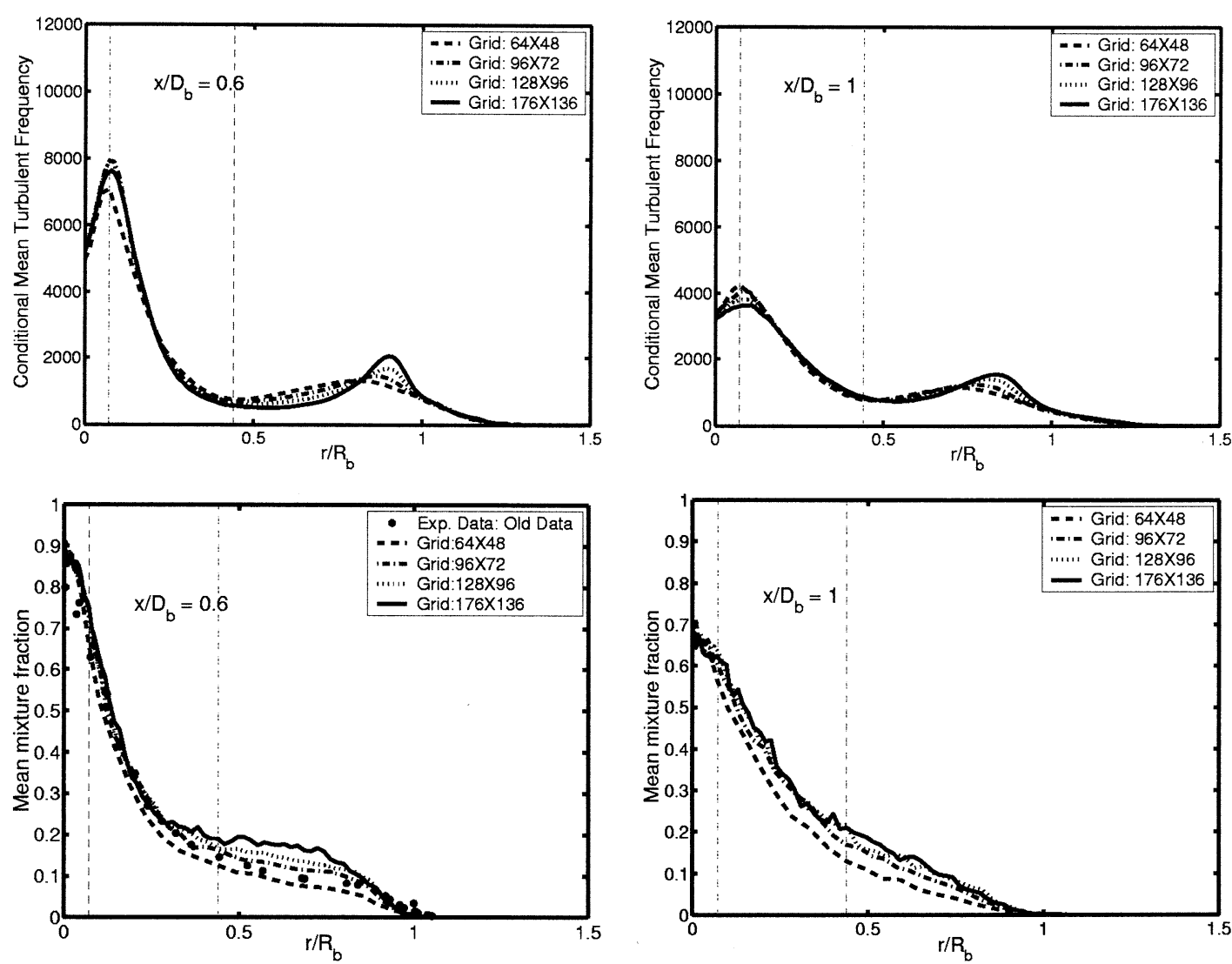

Fig. 14. Profiles of conditional mean turbulent frequency $(\Omega(1 / s))$ (top plots) and mean mixture fraction $(\tilde{\xi})$ (bottom plots) at the axial locations $0.6 D_{b}$ (left plots) and $D_{b}$ (right plots) computed on $176 \times 136,128 \times 96,96 \times 72$, and $64 \times 48$ grids. $N_{p c}=$ $100, N_{T A}^{P 2 P}=500$.

ity is first examined. For this purpose the time histories of the mean axial velocity and the mean turbulent kinetic energy are plotted in Fig. 12 at the selected observation locations (Table 2). The calculation is performed on a $176 \times 136$ grid with the number of particles per cell $N_{p c}=50$ and the time-averaging factor $N_{T A}^{P 2 P}=500$, which, as will be shown later, corresponds to the spatial and bias errors less than $5 \%$ in all the mean quantities. The figure shows that the statistical stationary state is attained after about 8000 particle time steps for this case.

\subsubsection{Grid convergence}

The grid convergence is examined and the spatial error is quantified in this section. The spatial error results from the spatial discretization in the $\mathrm{FV}$ method as well as from the mean field estimation, i.e., kernel estimation and interpolation schemes used in the particle algorithm due to the finite size of the mesh cells. The spatial error in the hybrid method has been extensively studied before [5,6], and it has been shown that the method is second order accurate in space. Therefore, an extensive study of spatial error is not repeated here and it is rather intended to show the grid convergence. For this purpose, the timeaveraged profiles of the mean velocity and the rms axial fluctuating velocity, and the conditional mean turbulent frequency and mean mixture fraction are plotted in Figs. 13 and 14, respectively, to show overall dependence of the calculated results on the grid refinement. The dotted vertical lines shown in Figs. 13 and 14 are drawn to mark the locations where the spatial error is quantified as will be discussed below. As can be seen in these figures, both $\tilde{U}$ and $u^{\prime}$ are very sensitive to the grid size indicating relatively large spatial error in these fields, especially in $u^{\prime}$ near the centerline. Fig. 14 indicates that the mean fields $\Omega$ and $\tilde{\xi}$ are also very sensitive to the grid size especially near the inlet plane behind the bluffbody but are not as sensitive as $\tilde{U}$ and $u^{\prime}$. To quantify the spatial error and to verify the second-order spatial accuracy of the hybrid method, the mean quantities $\tilde{U}, u^{\prime}, \Omega$, and $\tilde{\xi}$ are plotted against $M^{-2}$ in Fig. 15 at the selected locations specified in Table 3 . The sym- 

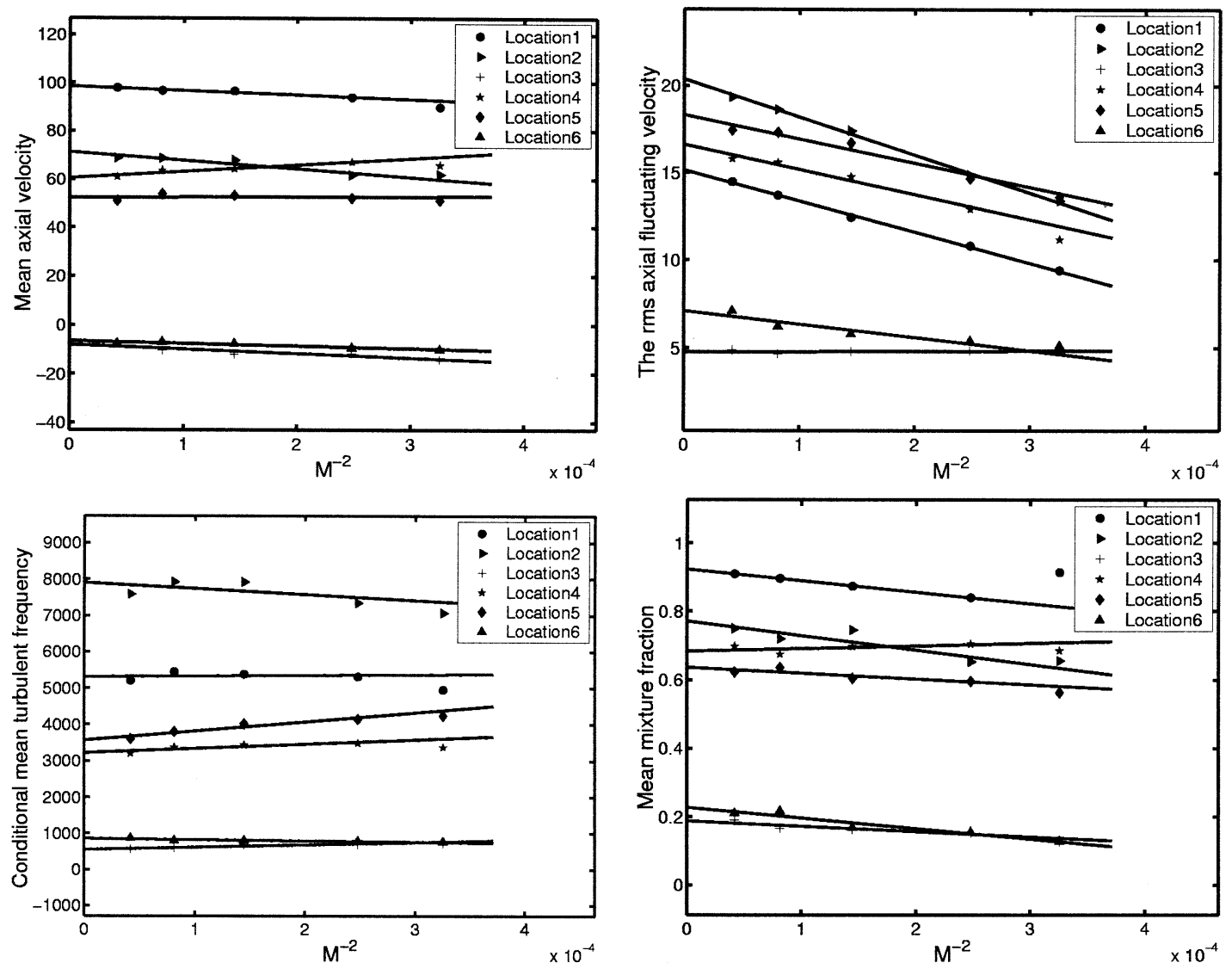

Fig. 15. Time-averaged mean quantities against the inverse of the total number of grid cells $M^{-2}$ at the selected locations (Table 3 ) showing the expected second order accuracy of the method. The solid lines are linear least-square fits to numerical data.

bols in Fig. 15 represent the numerical results and the lines are linear least-squares fits to the numerical data. The approximate linear relationship between the mean quantities and $M^{-2}$ confirms the expected second order accuracy. To quantify the spatial error and to determine the grid size required for a given error tolerance, we define the normalized spatial error for a mean quantity $Q$ as

Table 3

Six selected locations in the computational domain used to quantify spatial and bias errors; $D_{b}=50 \mathrm{~mm}$ is bluffbody diameter and $R_{j}=1.8 \mathrm{~mm}$ is jet radius

\begin{tabular}{lll}
\hline & $\begin{array}{l}\text { Axial } \\
\text { distance }(x)\end{array}$ & Radial distance $(r)$ \\
\hline Location 1 & $0.6 D_{b}$ & 0.0 \\
Location 2 & $0.6 D_{b}$ & $R_{j}$ \\
Location 3 & $0.6 D_{b}$ & mid bluff-body $=13.4 \mathrm{~mm}$ \\
Location 4 & $D_{b}$ & 0.0 \\
Location 5 & $D_{b}$ & $R_{j}$ \\
Location 6 & $D_{b}$ & mid bluff-body $=13.4 \mathrm{~mm}$ \\
\hline
\end{tabular}

$\epsilon_{s_{Q}}=\frac{\left|Q_{M, N_{p c}}-Q_{\infty, N_{p c}}\right|}{\max \left(\left|Q_{\infty, N_{p c}}\right|, \kappa_{s}\left|Q_{\mathrm{ref}}\right|\right)}$

where $Q_{M, N_{p c}}$ is the numerical result obtained using $M^{2}$ grid cells with $N_{p c}$ number of particles per cell, $Q_{\infty, N_{p c}}$ is the extrapolated value as $M \rightarrow \infty$ while keeping $N_{p c}$ constant, $Q_{\text {ref }}$ is the reference value taken as the largest value of $Q$ in magnitude at the inlet and $\kappa_{s}$ is a positive number taken here as 0.2. Based on Eq. 21, the approximate grid sizes required for a $5 \%$ error tolerance for the mean fields $\tilde{U}, u^{\prime}, \Omega$, and $\tilde{\xi}$ are calculated at the observation locations, and are shown in Table 4 . As can be seen in this table, a $176 \times 135$ (corresponding to $M^{-2}=4.21 \times 10^{-5}$ ) grid is sufficient to reduce the spatial error below $5 \%$ in all the mean quantities at these locations.

\subsubsection{Bias error}

Bias error is a deterministic error caused by using a finite number of particles and is expected to scale as $N_{p c}^{-1}$ [12]. To explore the bias error in different mean quantities, calculations are performed using different 
Table 4

Computational grid required for $5 \%$ spatial error tolerance

\begin{tabular}{llllllr}
\hline & $\tilde{U}$ & $u^{\prime}$ & $v^{\prime}$ & $\Omega$ & $\tilde{\xi}$ & $\xi^{\prime \prime}$ \\
\hline Location 1 & $70 \times 53$ & $176 \times 135$ & $158 \times 119$ & $48 \times 36$ & $32 \times 24$ & $100 \times 75$ \\
Location 2 & $99 \times 74$ & $170 \times 127$ & $175 \times 131$ & $69 \times 52$ & $75 \times 57$ & $111 \times 84$ \\
Location 3 & $71 \times 53$ & $19 \times 14$ & $59 \times 45$ & $51 \times 39$ & $48 \times 36$ & $103 \times 78$ \\
Location 4 & $87 \times 65$ & $138 \times 104$ & $147 \times 110$ & $77 \times 58$ & $65 \times 49$ & $48 \times 36$ \\
Location 5 & $19 \times 14$ & $136 \times 102$ & $148 \times 111$ & $99 \times 74$ & $93 \times 70$ & $71 \times 53$ \\
Location 6 & $54 \times 40$ & $100 \times 75$ & $104 \times 78$ & $19 \times 14$ & $34 \times 26$ & $95 \times 71$ \\
\hline
\end{tabular}

number of particles per cell ranging from $N_{p c}=20$ to $N_{p c}=160$ on a $128 \times 96$ grid. The radial profiles calculated with various values of $N_{p c}$ are plotted for $\tilde{U}$ and $u^{\prime}$ in Fig. 16 and for $\Omega$ and $\tilde{\xi}$ in Fig. 17 to show the overall variations of the bias error in these quantities. The dotted vertical lines shown in Figs. 16 and 17 are again drawn to mark the locations where the bias error is quantified as will be discussed below. The small differences between the radial profiles in- dicate very small bias error in all the mean quantities as being consistent with the earlier results obtained with the hybrid method [5,6]. To quantify the bias error and to verify the expected scaling with $N_{p c}$, the mean quantities $\tilde{U}, u^{\prime}, \Omega$, and $\tilde{\xi}$ are plotted against $N_{p c}^{-1}$ in Fig. 18 at the selected locations (Table 3). In Fig. 18, the symbols represent the numerical calculations and the solid lines are the linear least squares fits to the numerical data. The approximate linear
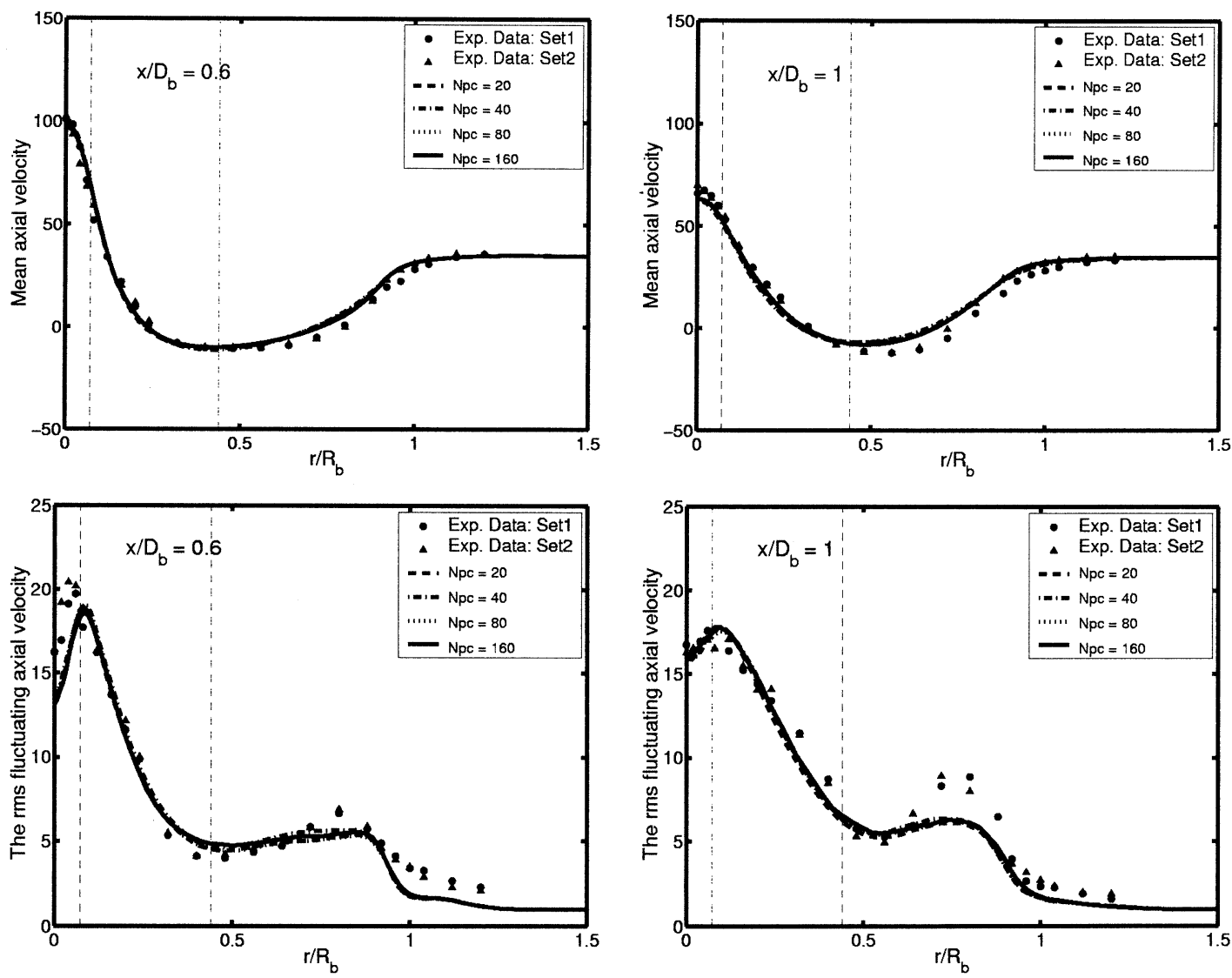

Fig. 16. Profiles of mean axial velocity $(\tilde{U}(\mathrm{~m} / \mathrm{s}))$ (top plots) and the rms fluctuating axial velocity $\left(u^{\prime}(\mathrm{m} / \mathrm{s})\right.$ ) (bottom plots) at the axial locations $0.6 D_{b}$ (left plots) and $D_{b}$ (right plots) computed on $128 \times 96$ grid and $N_{p c}$ ranges from 20 to 160 . $N_{T A}^{P 2 P}$ $=500$. 

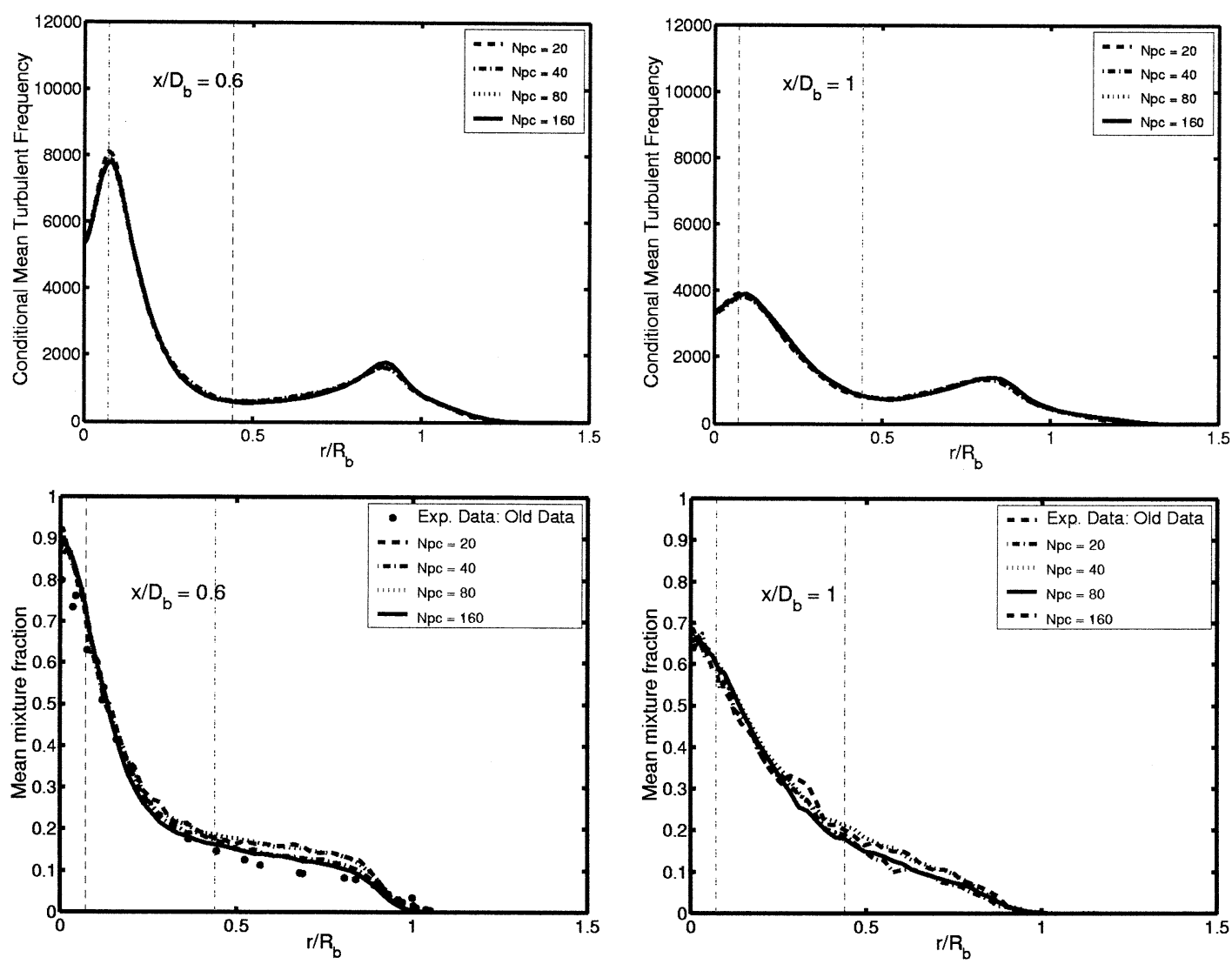

Fig. 17. Profiles of conditional mean turbulent frequency $(\Omega(1 / s))$ (top plots) and mean mixture fraction $(\tilde{\xi})$ (bottom plots) at the axial locations $0.6 D_{b}$ (left plots) and $D_{b}$ (right plots) computed on $128 \times 96$ grid and $N_{p c}$ ranges from 20 to $160 . N_{T A}^{P 2 P}$ $=500$.

relationship between the mean quantities and $N_{p c}^{-1}$ confirms the expected scaling of the bias error. Similar to the normalized spatial error, the normalized bias error for a mean quantity $Q$ is defined as

$\epsilon_{b_{Q}}=\frac{\left|Q_{M, N_{p c}}-Q_{M, \infty}\right|}{\max \left(\left|Q_{M, \infty}\right|, \kappa_{b}\left|Q_{\text {ref }}\right|\right)}$

where $Q_{M, N_{p c}}$ is the numerical result obtained using $N_{p c}$ number of particles per cell with $M^{2}$ grid cells, $Q_{M, \infty}$ is the extrapolated value as $N_{p c} \rightarrow \infty$ while keeping the grid size the same, $Q_{\text {ref }}$ is the same reference value as used in Eq. 21 for the spatial error and $\kappa_{b}$ is a positive number taken as 0.2 . Table 5 shows the minimum number of particles per cell needed to reduce the bias error below 5\% error tolerance for the mean quantities $\tilde{U}, u^{\prime}, \Omega$, and $\tilde{\xi}$ at the selected locations. As can be seen in this table, the largest bias error occurs in $\Omega$ and about 30 particles per cell are sufficient to have the bias error less than $5 \%$ in all the mean quantities at these locations.

\section{Conclusions}

Computations using a velocity-turbulent frequency-compositions joint PDF method combined with a consistent hybrid $\mathrm{FV} /$ particle solution algorithm are reported for a bluff-body stabilized turbulent flame. A simple flamelet chemistry model is used in the present study and it is intended to form the foundations for the future PDF calculations of the bluffbody flames with detailed chemistry models. Hence the focus here is placed on the accurate calculations of the mean flow, turbulence and mixing.

It is demonstrated that the method is convergent in terms of reaching a statistically stationary state and also in terms of grid refinement and particle numbers. A $176 \times 136$ grid and 30 particles per cell are found to be sufficient to reduce the spatial and bias errors below a $5 \%$ error tolerance. The performance of the joint PDF method is assessed in terms of predicting the flow and scalar mean fields by comparing the computed radial mean profiles with the available ex- 

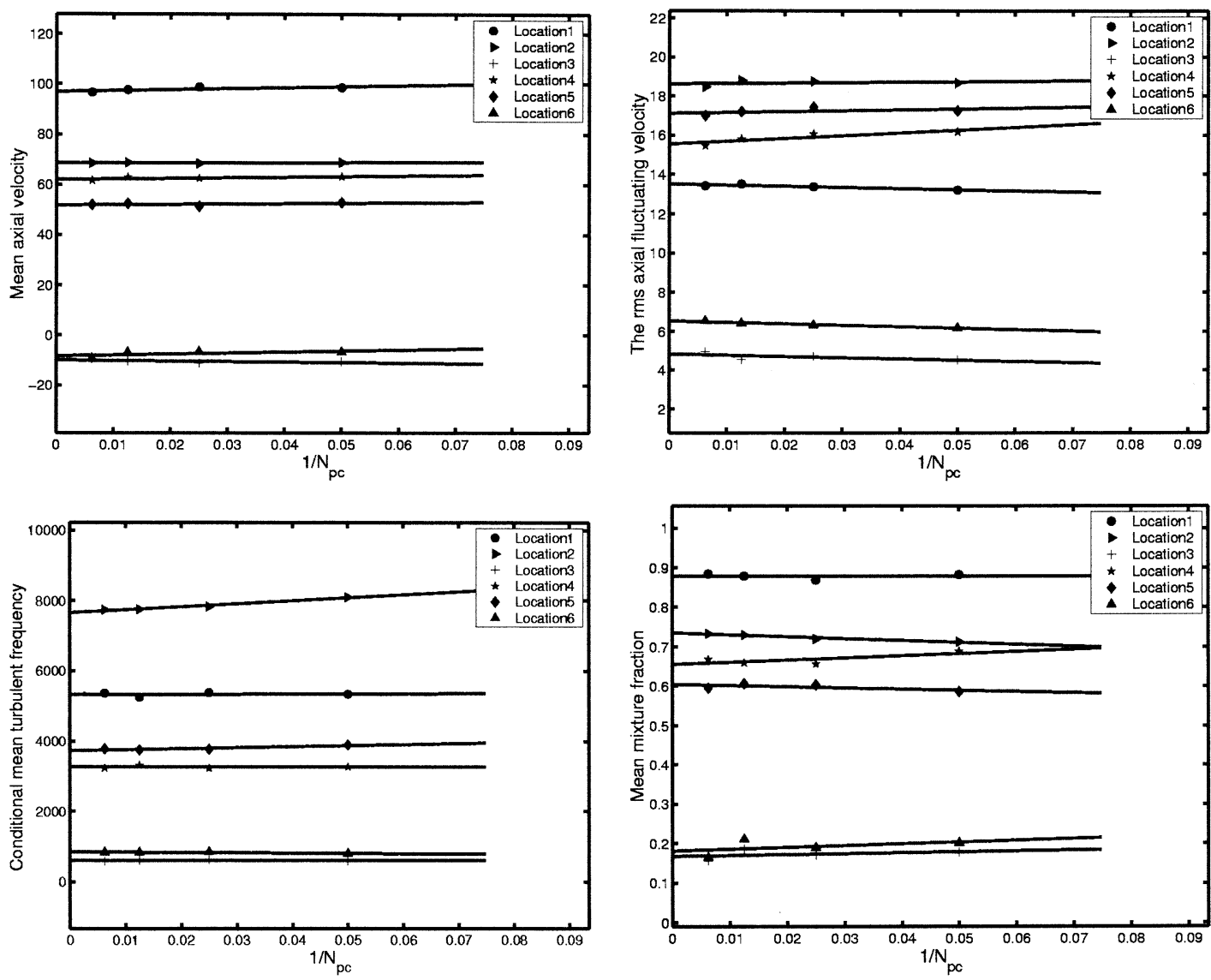

Fig. 18. Time-averaged mean quantities against $N_{p c}^{-1}$ at the selected locations (Table 3) showing the expected scaling for bias error. The solid lines are linear least-square fits to numerical data.

perimental data. Considering the simplest velocity, mixing and chemistry models are used in the computations, the PDF method predicts both the flow and scalar mean fields very well especially within the recirculation zone.

The sensitivities of the computational results to the model constants $C_{\omega 1}$ in the turbulent frequency model and $C_{\phi}$ in the mixing model are also investi-

Table 5

Number of particles per cell $\left(N_{p c}\right)$ required for a $5 \%$ bias error tolerance

\begin{tabular}{llrlrrr}
\hline Mean fields & $\tilde{U}$ & $u^{\prime}$ & $v^{\prime}$ & $\Omega$ & $\tilde{\xi}$ & $\xi^{\prime \prime}$ \\
\hline Location 1 & 9 & 9 & 9 & 29 & 3 & 1 \\
Location 2 & 2 & 3 & 3 & 30 & 23 & 10 \\
Location 3 & 5 & 7 & 4 & 3 & 1 & 5 \\
Location 4 & 6 & 16 & 4 & 13 & 1 & 13 \\
Location 5 & 4 & 5 & 7 & 12 & 8 & 7 \\
Location 6 & 9 & 8 & 8 & 1 & 3 & 11
\end{tabular}

gated. It is found that all the mean quantities but especially the mean turbulent frequency and the rms fluctuating velocity are very sensitive to the changes in $C_{\omega 1}$. It is observed that the mixing model constant $C_{\phi}$ has a strong influence on the rms fluctuating mixture fraction but not much influence on the mean mixture fraction and the mean flow quantities.

It is found that about $40 \mathrm{CPU}$ hours are needed to carry out a PDF simulation for the present bluff-body flame with the spatial and bias errors less than 5\% in the all mean quantities on a $500 \mathrm{Mhz}$ single processor PIII PC.

\section{Acknowledgments}

This work is supported in part by Department of Energy, Grant number DE-FG02-90ER 14128 and in part by DE-AC26-00NT40847. 


\section{References}

[1] S.B. Pope, Annu. Rev. Fluid. Mech. 26 (1994) 23-63.

[2] P. Jenny, M. Muradoglu, K. Liu, S.B. Pope, D.A. Caughey, J. Comp. Phys. 166 (2001) 1-23.

[3] M.S. Anand, A.T. Hsu, S.B. Pope, AIAA J. 35 (1997) 1143-1150.

[4] S.B. Pope, Prog. Energy Combust. Sci. 11 (1985) 119-192.

[5] M. Muradoglu, P. Jenny, S.B. Pope, D.A. Caughey, J. Comp. Phys. 154 (1999) 342-371.

[6] M. Muradoglu, S.B. Pope, D.A. Caughey, J. Comp. Phys. 172 (2001) 841-878.

[7] M. Muradoglu, S.B. Pope, AIAA J. 40 (2002) 17551763.

[8] P.R. Van Slooten, Jayesh, S.B. Pope, Phys. Fluids 10 (1998) 246-265.

[9] B.B. Dally, D.F. Fletcher, A.R. Masri, Combust. Theory and Modelling 2 (1998) 193-219.

[10] P. Kalt, A.R. Masri, Private Communication, 2000.

[11] B.B. Dally, A.R. Masri, R.S. Barlow, G.J. Fiechtner, Combust. Flame 114 (1998) 119-148.

[12] J. Xu, S.B. Pope, J. Comput. Phys. 152 (1998) 246265.

[13] Q. Tang, J. Xu, S.B. Pope, Proc. Combust. Inst. 28 (2000) 133-139.

[14] S.H. Kim, K.Y. Huh, Fifth International Workshop on Measurement and Computation of Turbulent Nonpremixed Flames (TNF5), Technical University of Delft, The Netherlands (2000).

[15] B. Merci, D. Roekaerts, T.W.J. Peters, E. Dick, J. Vierendeels, Combust. Flame 126 (2001) 1533-1556.

[16] H.A. Wouters, P.A. Nooren, T.W. Peeters, D. Roekaerts, Twenty-Sixth Symposium (International) on
Combustion, The Combustion Institute, Pittsburgh, 1996, p. 246.

[17] S.H. Kim, K.Y. Huh, L. Tao, Combust. Flame 120 (2000) 75-90.

[18] S.M. Correa, S.B. Pope, Twenty-Fourth Symposium (International) on Combustion, The Combustion Institute, Pittsburgh, 1996, pp. 279-285.

[19] P. Koutmos, P. Marazioti, Int. J. Numer. Meth. Fluids 35 (2001) 939-959.

[20] N. Peters, Prog. Energy Combust. Sci. 10 (1984) 319339.

[21] GRI Mechanism 2.1, available from the World Wide Web site http://www.gri.org.

[22] R.W. Bilger, S.H. Starner, R.J. Kee, Combust. Flame 80 (1990) 135-149.

[23] C. Dopazo, Recent developments in PDF methods, in: P.A. Libby, F.A. Williams (Eds.), Turbulent Reacting Flows, Academic Press, London, 1994, p. 375.

[24] P. Jenny, S.B. Pope, M. Muradoglu, D.A. Caughey, J. Comp. Phys. 166 (2001) 218-252.

[25] S.B. Pope, PDF2DV: A Fortran code to solve the modeled joint PDF equations for two-dimensional turbulent flows, Internal report, Cornell University, Ithaca, NY, 1994.

[26] D.A. Caughey, AIAA J. 26 (1988) 841-851.

[27] H. Mobus, P. Gerlinger, D. Bruggemann, Combust. Flame 124 (2001) 519-534.

[28] A.R. Masri, R.W. Dibble, R.S. Barlow, Prog. Energy Combust. Sci. 22 (1996) 307-362.

[29] A.R. Masri, Technical report, Dept. of Mechanical and Mechatronic Engr., The University of Sydney, Australia, available at: http://www.mech.eng.usyd.edu.au/ research/energy/.

[30] S.B. Pope, Turbulent Flows, Cambridge University Press, Cambridge, UK, 2000. 